\title{
Feedback control for form-drag reduction on a bluff body with a blunt trailing edge
}

\author{
Jeremy A. Dahan ${ }^{1} \dagger$, A. S. Morgans ${ }^{1}$ and S. Lardeau ${ }^{2}$ \\ ${ }^{1}$ Department of Aeronautics, Imperial College London, London SW7 2AZ, UK \\ ${ }^{2}$ CD-adapco, 200 Shepherds Bush Road, London W6 7NL, UK \\ (Received 3 October 2011; revised 21 March 2012; accepted 23 May 2012; \\ first published online 3 July 2012)
}

The objective of this numerical study is to increase the base pressure on a backwardfacing step via linear feedback control, to be ultimately translated to a drag reduction on a blunt-based bluff body. Two backward-facing step cases are simulated: a laminar two-dimensional (2D) flow at a Reynolds number of $R e_{\theta}=280$, and a turbulent three-dimensional (3D) flow at $R e_{\theta}=1500$ using large-eddy simulation. The control is effected by a full-span slot jet with zero-net-mass-flux, and two jet locations are examined. Linear system identification is performed to characterize the flow response to actuation, used to synthesize a control law. The control strategy is based on the premise that an attenuation of the instantaneous pressure fluctuations on the base of the step should lead to an increase in the time-averaged base pressure. Open-loop harmonic forcing is examined within a broad frequency range for both the 2D and 3D flows, which are found to respond differently to actuation. The controllers based on disturbance attenuation lead to sensible increases in base pressure (up to $70 \%$ in $2 \mathrm{D}$ and $20 \%$ in 3D) with higher efficiency than the best results achieved in open-loop. The results support the conjecture about the link between the base pressure fluctuations and mean, although it is shown that such a black-box model approach is not suitable for optimization without further physical insight.

Key words: control theory, drag reduction, shear layers

\section{Introduction}

For flows around bluff bodies with a blunt trailing edge, flow separation is fixed at the trailing edge. This flow separation leads to an unsteady wake and high form drag. The ability to reduce form drag by controlling the wake is highly pertinent, both in terms of physical understanding of the control impact and with respect to industrial applications. A prominent example where this knowledge could translate to economic and environmental benefits is road transportation, where form drag is a dominant source of energy loss at high speeds (Seifert et al. 2009).

One may distinguish three types of control actions: passive, active open-loop or active closed-loop (feedback) control. Passive control, sometimes referred to as flow management (Gad-el Hak, Pollard \& Bonnet 1998), uses actuation without power input. Passive control for form-drag reduction has been extensively studied. It is well known that addition of a splitter plate along the wake centreline is an efficient means to delay vortex shedding and increase the base pressure (Roshko 1954). Tanner (1972)

$\dagger$ Email address for correspondence: jeremy.dahan05@imperial.ac.uk 
investigated the flow behind a wing with a blunt trailing edge. He introduced a broken separation line using a segmented trailing edge and measured drag reductions of up to $64 \%$. More recently, Park et al. (2006) found that adding small tabs to the upper and lower trailing edges of a blunt body yields significant drag reductions. A few passive devices have been tested on full-scale road vehicles. For instance, Modi, Hill \& Yokomizo (1995) installed trip fences on the front face of a truck trailer and obtained drag reductions of up to $16 \%$.

However, passive devices may have adverse effects away from their design point. Open-loop control (corresponding to powered actuation without sensing) can reproduce the beneficial effects of passive devices and widen the operating range. Wood (1967) notably showed that base bleed displaces the vortex formation region further downstream from the trailing edge of a two-dimensional bluff body, resulting in base pressure increase. Extensive wind-tunnel testing on truck models by Seifert et al. (2009) has shown open-loop control to be capable of net fuel reductions exceeding $10 \%$. Englar (2000) also achieved significant drag reduction for model trucks and streamlined vehicles using circulation control.

Finally, closed-loop control is achieved via powered actuators responding to sensors in the flow field. In contrast to open-loop actuation, feedback control can modify the dynamics of a system, for instance stabilizing flows with unstable modes such as cavity resonances (Cattafesta et al. 2008) or thermoacoustic instabilities (Dowling \& Morgans 2005). In addition, closed-loop control offers further degrees of freedom to deal with uncertainty and increase efficiency. Feedback control strategies for drag reduction of bluff bodies are usually categorized into separation control or direct wake control. The former apply only to bodies with moveable separation points such as the circular cylinder (Siegel, Cohen \& McLaughlin 2006) or a step with a rounded edge (Kim et al. 2006). Significantly less work has been carried out on using direct wake control to reduce form drag on bluff bodies with a blunt trailing edge, although a few examples do exist. For instance, Henning \& King (2005) used quantitative feedback theory to increase the base pressure of a D-shaped wind-tunnel model. Pastoor et al. (2008) also examined feedback strategies for drag reduction on the same bluff body and achieved a $15 \%$ drag reduction. Stalnov, Fono \& Seifert (2011) performed an experimental investigation aimed at stabilizing the wake of a D-shaped bluff body with a proportional-integral control law. They showed that their controller leads to a concomitant drag reduction associated with a delayed roll-up of the separating shear layers, hence a reduction in the streamwise momentum transferred to the recirculation region.

The objective of the present study is to investigate numerically the use of feedback control to achieve a sensible form-drag reduction on a blunt-based bluff body, truncated to a backward-facing step for computational economy. A simplified twodimensional (2D) problem is first approached, after which the flow on a fully turbulent three-dimensional (3D) step is examined. In $\S 2$, a short review of the backward-facing step flow is presented, as well as the computational details and a description of the feedback control design method. The $2 \mathrm{D}$ and $3 \mathrm{D}$ cases are respectively studied in $\S \S 3$ and 4 , before finishing with concluding remarks in $\S 5$.

\section{Problem formulation}

The geometry considered is presented in figure 1, representing a bluff body with a blunt trailing edge. Significant computational savings are obtained by focusing on the region of interest: the wake. Thus, only the downstream part of the flow 


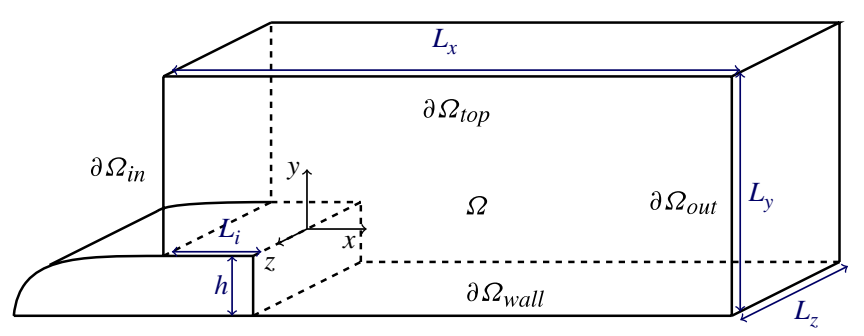

FIGURE 1. Diagram of the computational domain $\Omega$. The flow is from left to right. Dimensions not to scale.

field is computed, and the flow domain reduces to a backward-facing step. A careful investigation comparing the full-domain and truncated flows (without actuation) validated this approach, provided a realistic inflow boundary condition is used. A brief overview of the backward-facing step (BFS) flow and attempts to control it is now given.

\subsection{Backward-facing step flow}

The unsteady flow downstream of a step may be roughly divided into four regimes: a growing shear layer, a recirculation bubble with backflow velocities exceeding $0.2 U_{0}$ (where $U_{0}$ is the free-stream velocity upstream of the step), a reattachment region and a redeveloping boundary layer after reattachment (Henning \& King 2007). The separated shear layer appears similar to a plane mixing layer up to $x / h \approx 3$, where $h$ is the step height, except for the high level of turbulence on the low-speed side (Simpson 1989). As the shear layer grows, the presence of the wall becomes influential and the flow reattaches.

Bradshaw \& Wong (1972) argued that the shear layer splits in the reattachment region, with a part being deflected into the separation bubble and supplying entrainment upstream. However, there is no experimental evidence of large-scale structures being swept upstream. Eaton \& Johnston (1981) noted two important properties of the reattaching shear layer. They first observed that the Reynolds stresses drop abruptly in the reattachment region and secondly, that the reattached layer is very slow to readjust to a conventional turbulent boundary layer profile. It has been conjectured that the first characteristic is associated with a halt in vortex pairing induced by the wall, while the second is caused by the presence of vortical structures produced in the separated shear layer that survive for a long downstream distance (Troutt, Scheelke \& Norman 1984). Indeed, coherent structures formed in the separated shear layer roll up and pair, which makes the shear layer grow and generates high Reynolds stresses, as in a free mixing layer. The presence of the wall in the BFS flow, however, interrupts this mechanism in the reattachment region.

A number of studies have focused on active control of the BFS flow. While steady suction or blowing have been considered as control methods to reduce the size of the separated bubble (e.g. Uruba, Jonáš \& Mazur 2007), pulsating actuation resonating with flow instabilities is recognized as more efficient. Bhattacharjee, Scheelke \& Troutt (1986), Hasan \& Khan (1992), Chun \& Sung (1996) and Wengle et al. (2001) have reported the maximum reduction of the reattachment length when perturbing the flow with a Strouhal number $S t_{h}=f h / U_{0} \approx 0.2$, where $f$ is the actuation frequency. 
At least three instability modes are influential in reattaching shear layers: the shear layer, the shedding (or step) and the flapping modes (Leschziner \& Lardeau 2011). Hasan \& Khan (1992) found that the shear layer mode, linked to Kelvin-Helmholtztype instabilities, scales with the boundary layer momentum thickness at separation, $\theta$, and is characterized by $S t_{\theta}=0.011$ for a turbulent separation. In addition, they suggested that the shear layer mode reduces to the step mode through vortex pairing. The step mode is generally recognized to be characterized by $S t_{h} \approx 0.2$. Finally, the flapping mode corresponds to a global motion of the separation bubble, and its frequency is expected to be one order of magnitude lower than the shedding mode.

\subsection{Numerical simulations}

This work employs an in-house large-eddy simulation (LES) code, 'stream-LES', to solve the incompressible Navier-Stokes equations. The code was developed by Lardat \& Leschziner (1998) and a detailed description and validation can be found in Temmerman (2004). The procedure is based on a general non-orthogonal grid, blockstructured, finite-volume method with a fully collocated storage. Pressure-velocity decoupling, arising from the collocated formulation, is counteracted by the Rhie and Chow interpolation practice.

The spatial derivatives are transformed to surface integrals using the Gaussdivergence theorem and evaluated with quadrature formulae, yielding a secondorder spatial discretization. Time-marching is based on a fractional step method incorporating third-order approximations for the convection and diffusion terms and a third-order Gear-like scheme for the time derivative, shown to possess advantageous stability over a corresponding second-order time-advancement scheme (Fishpool \& Leschziner 2009). The first step consists of solving the momentum equation without the pressure term. An intermediate velocity field is obtained that does not satisfy the continuity equation. This is corrected via the pressure gradient by projection onto a divergence-free vector field. The implicit Poisson problem for the pressure is solved by means of a partial diagonalization technique and a $2 \mathrm{D} \mathrm{V}$-cycle multigrid method combined with a successive line overrelaxation algorithm. The subgrid-scale stresses are simulated by the wall-adapting local eddy-viscosity (WALE) model proposed by Nicoud \& Ducros (1999). The WALE model reproduces the cubic wall-asymptotic behaviour of the eddy viscosity and returns lower values of this viscosity compared to the Smagorinsky model (Temmerman 2004).

Two distinct flow cases, both relating to figure 1, will be considered in this work: a $2 \mathrm{D} \operatorname{BFS}\left(L_{z} / h=0\right)$ with a laminar inflow boundary layer and a $3 \mathrm{D} \operatorname{BFS}\left(L_{z} / h=4\right)$ with a turbulent inflow boundary layer. Since the former case is laminar and the latter is turbulent, one expects significant differences between the two flows. The aim of examining these two cases is to show that a single feedback control strategy may apply to both. The boundaries of the domains $\Omega_{2 D}$ and $\Omega_{3 D}$ are decomposed into $\partial \Omega=\partial \Omega_{\text {in }} \cup \partial \Omega_{\text {wall }} \cup \partial \Omega_{\text {top }} \cup \partial \Omega_{\text {out }}$, where $\partial \Omega_{\text {in }}$ is the inflow boundary, $\partial \Omega_{\text {wall }}$ represent the lower surfaces and the step face modelled with no-slip, $\partial \Omega_{\text {top }}$ is the upper surface set with a free-slip condition and $\partial \Omega_{\text {out }}$ is the outflow boundary where an advection equation for the velocity is imposed. For $\Omega_{3 D}$, spanwise periodic conditions are imposed on the sidewalls. The dimensions of $\Omega_{2 D}$ and $\Omega_{3 D}$ were chosen so as to avoid errors related to a constricted computational domain. The $2 \mathrm{D}$ flow was found to be more sensitive to confinement due to the free-slip condition and to the inlet length $L_{i}$. Therefore $\Omega_{2 D}$ and $\Omega_{3 D}$ have different $L_{i}$ and $L_{y}$ dimensions. For $\Omega_{2 D}$, $\left(L_{i}, L_{x}, L_{y}, L_{z}\right)=(4 h, 24 h, 9 h, 0)$ and for $\Omega_{3 D},\left(L_{i}, L_{x}, L_{y}, L_{z}\right)=(2 h, 14 h, 3 h, 4 h)$. The influence of the spanwise extent of $\Omega_{3 D}$ on the flow behaviour is discussed in $\S 4$. 


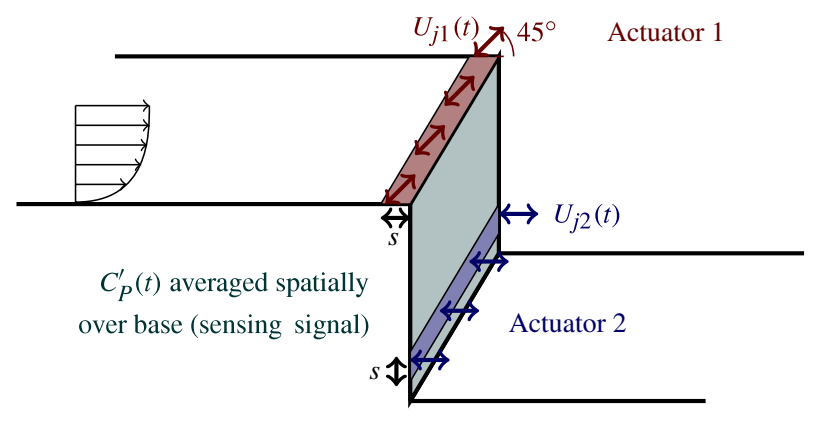

FIgURE 2. (Colour online) Schematic of the two actuator locations. Actuator 1 is near separation and actuator 2 is close to the step foot (targeting a secondary recirculation bubble).

In the 2D case, no subgrid-scale model is used because the flow is laminar; the simulation is akin to a direct numerical simulation (DNS). A Blasius boundary layer profile of thickness $\delta_{99}=h$ with a free-stream velocity $U_{0}$ is imposed on $\partial \Omega_{i n}$. The Reynolds number is set to $R e_{h}=U_{0} h / v=2000$, corresponding to $\operatorname{Re}_{\theta}=280$, where $v$ is the kinematic viscosity. In terms of the grid, $2.5 \times 10^{4}$ computational cells, with local refinement in the step and injection regions, were found to suffice, after extensive grid resolution checks.

The 3D case, on the other hand, has a fully turbulent inlet boundary layer with $\delta_{99}=0.5 h$ and $R e_{\theta}=1500$. The inlet conditions are generated by a precursor simulation for a boundary layer developing along a flat plate. The precursor simulation uses the recycling method of Lund, Wu \& Squires (1998). The 3D mesh contains $6 \times 10^{6}$ cells. Near the wall, the grid resolution in wall-units is $\Delta y_{\min }^{+}=0.85$ and the spanwise spacing is $\Delta z^{+}=18$, with the wall-unit scaling based on the inlet friction velocity $u_{\tau}$. The grid expansion ratio is limited to 1.05. A much finer grid composed of $28 \times 10^{6}$ computational cells, hereafter called the fine grid, is used to check the results for the baseline flow and a few selected cases of open-loop forcing.

\subsection{System identification and feedback controller design}

A fundamental consideration in any feedback control strategy is the selection of the actuation-sensing couple. Once this parameter is fixed, various routes are possible. Here we choose to implement a model-based feedback. Thus a model of the process to be controlled is sought before synthesizing the controller.

There are a number of actuator types in use in flow control (see Cattafesta \& Sheplak 2011). In the present work, zero-net-mass-flux slot jets are selected. For both the laminar and turbulent flows, two actuator configurations are investigated. The first slot jet actuator is located just upstream of the step corner and injected at an angle of $45^{\circ}$ whilst the second actuator is placed near the step foot and injected in the streamwise direction, as sketched in figure 2. Both actuators extend along the entire domain span and have a slot width $s=0.03 h$. The first slot jet is located over $(-0.03 h \leqslant x \leqslant 0, y=0)$ and the second slot jet is located over $(x=0,-0.94 h \leqslant y \leqslant-0.91 h)$. The modelling of the actuators is kept simplistic and the jet cavity is not resolved. The discharge conditions are instead described by an imposed mass flux at the jet orifice with a top-hat spatial velocity profile. This is a numerical simplification which is widely used in the literature (see e.g. Dejoan \& Leschziner 2004; Kim et al. 2004) and it has been shown that the details of the cavity and the slot are less critical for slot jets than for round jets (Leschziner \& 

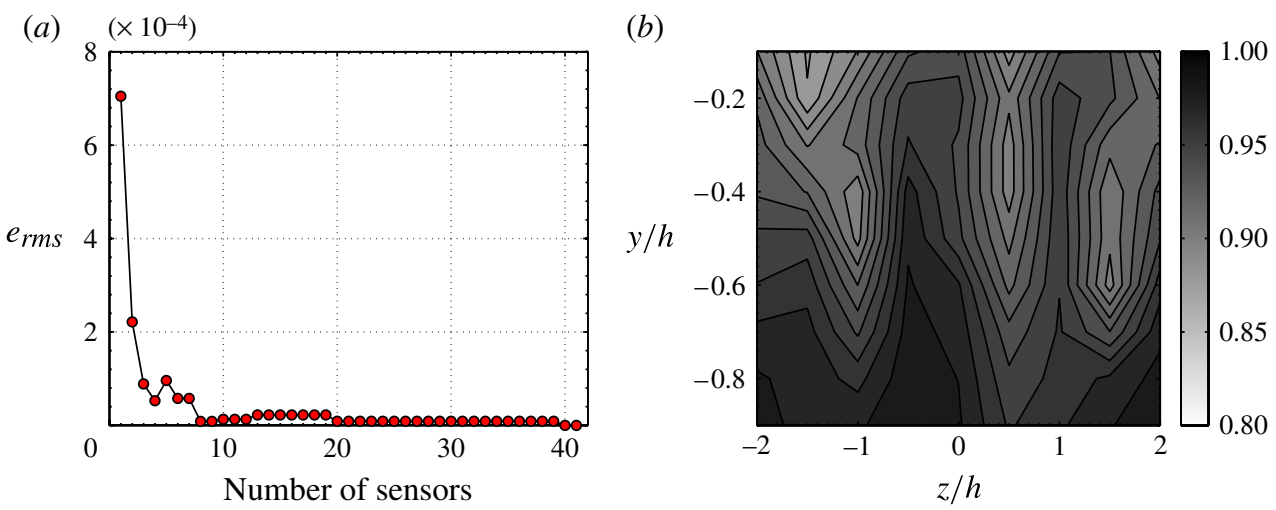

FIGURE 3. (Colour online) (a) Root-mean square of the error $e(t)=y(t)-y_{n}(t)$ against number of sensors, $n$, for the baseline flow in $\Omega_{2 D}$. Base pressure averaged continuously in space is denoted by $y(t)$, and $y_{n}(t)$ is the pressure signal averaged over $n$ uniformly spread sensors on the base. (b) Map of cross-correlation coefficient, for baseline flow in $\Omega_{3 D}$ between $y(t)$ and single-point pressure signal located at a given $(0, y, z)$ position on the base.

Lardeau 2011). The effects of the jet cavity can be modelled using more sophisticated velocity profiles (Aram, Mittal \& Cattafesta 2010) but this is not considered here. The actuation amplitude $A_{j}$ is characterized by the maximum ejection velocity at the slot if the actuation signal is periodic. If the actuation signal is not perfectly periodic, as is to be expected with closed-loop control, the actuation amplitude is time-dependent and equal to the slot velocity $A_{j}(t)=U_{j}(t)$, where $t$ is time non-dimensionalized by $U_{0} / h$.

The choice of the sensing arrangement - including the measured quantity, the number of sensors and their location - depends on the control objectives and on physical constraints. A heuristic method based on proper orthogonal decomposition (POD) was introduced by Cohen, Siegel \& McLaughlin (2006) to find an efficient configuration sufficient for closed-loop control. Here, an intuition-based approach is followed. Body-mounted sensors are preferred to sensors in the flow field, because they are more convenient for real-time control in experiments and practical applications. The sensor measurement should correlate with the target flow quantity, the mean base pressure, which is to be increased so as to reduce the form drag. Investigations of flow control on blunt-based bluff bodies indicate that a reduction in the amplitude of the base pressure fluctuations is associated with a time-mean base pressure increase (see Heenan \& Morrison 1998; Qubain 2009). The present feedback control approach utilizes this finding and aims to attenuate the fluctuations in base pressure with a view to consequentially increasing the mean base pressure. Hence, the sensor signal $y(t)$ used as input to the controller is the instantaneous base pressure fluctuations averaged spatially over the step base area, highlighted in figure 2 . Note that the choice of a zero-mean sensor signal is convenient since it will automatically lead to zero-net-mass-flux actuation. The spatial averaging is done continuously (using all the computational nodes covering the base). In practical applications, however, discrete pressure transducers are used. It is thus important to examine the impact of this averaging. Figure 3(a) shows the evolution, for the baseline flow in $\Omega_{2 D}$, of the root-mean-square of the error $e(t)=y(t)-y_{n}(t)$, with $n$ the number of sensors used for averaging ( $y_{n}$ denotes the signal obtained by averaging pressure with $n$ sensors). Using 
(a)

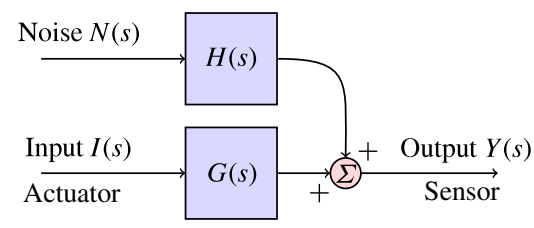

(b)

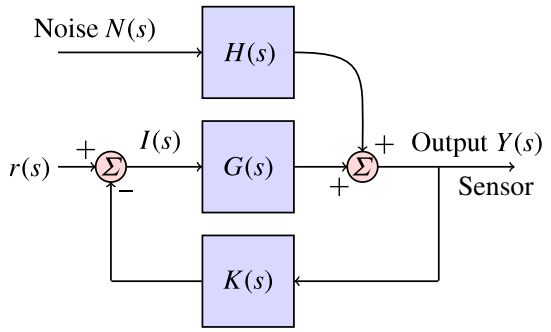

FIGURE 4. (Colour online) Frequency-domain models for: ( $a$ ) the open-loop control system; and $(b)$ the closed-loop control system.

a single sensor at the centre of the base results in a small error, which falls sharply if more points are used. The continuous average is recovered by using only 8 sensors. For the flow in the 3D domain $\Omega_{3 D}$, figure 3(b) plots contours of the cross-correlation coefficient between the continuous average and single-point sensors on the base. The correlations are everywhere very high, which means the continuous average does not hide any important localized structures. Furthermore, it implies that the flow near the base does not see significant 3D effects; this provides support for the use of $2 \mathrm{D}$ actuation.

The models used for the feedback controller design are sketched in figure 4. They assume that the fluctuations in the base pressure are caused both by actuation and by disturbances present in the uncontrolled flow. The latter are collectively represented as noise. $I(s), N(s)$ and $Y(s)$ denote respectively the actuation input, noise and plant output (sensor) signals. Note that $s$ is the complex Laplace transform variable. The input $I(s)$ is the Laplace transform of the actuation signal $U_{j}(t)=A_{j} \sin \left(2 \pi S t_{h} t\right)$, where $S t_{h}$ is the non-dimensional forcing frequency. The noise represents the upstream flow structures which affect the output signal. Finally, the output $Y(s)$ is the Laplace transform of $y(t)=1 / S_{b} \int_{S_{b}} C_{P}^{\prime}(t) \mathrm{d} S$, where $C_{P}^{\prime}$ is the fluctuating part of the pressure coefficient (based on free-stream velocity and pressure) $C_{P}=2\left(P-P_{0}\right) /\left(\rho U_{0}^{2}\right)$ and $S_{b}$ is the area of the base. $G(s)$ and $H(s)$ are the unknown transfer functions from the forcing input and the noise to the output, respectively.

Although $H(s)$ does not need to be explicitly evaluated, the design of a feedback controller, $K(s)$, requires a low-order model for $G(s)$. Assuming that the forcing amplitude is sufficiently small for the flow response to be dynamically linear (this assumption is later checked), this can be achieved via linear system identification (see Ljung 1999). Dynamic linearity implies that sinusoidal input modes are present in the sensor measurement with a gain and phase shift. The latter two may be measured from the LES and they define the frequency response $G(\mathrm{i} \omega)$ as a function of the angular frequency $\omega=2 \pi S t_{h}$.

There exist a wide variety of input signals available for system identification. One may, for example, actuate the flow harmonically at various frequencies and measure the gain and phase shift from the sensor output in each case. The main drawback of this approach is that each calculation yields information for a single frequency, so that many computations are required. Although a more economical solution is to select an input signal that contains a range of frequencies, such as a finite-time impulse or a sum-of-sines signal, harmonic forcing holds two important advantages: it allows one to derive physical insight into the effect of the forcing (at specific frequencies) on various aspects of the flow and it also allows weak nonlinearities to be characterized via the 

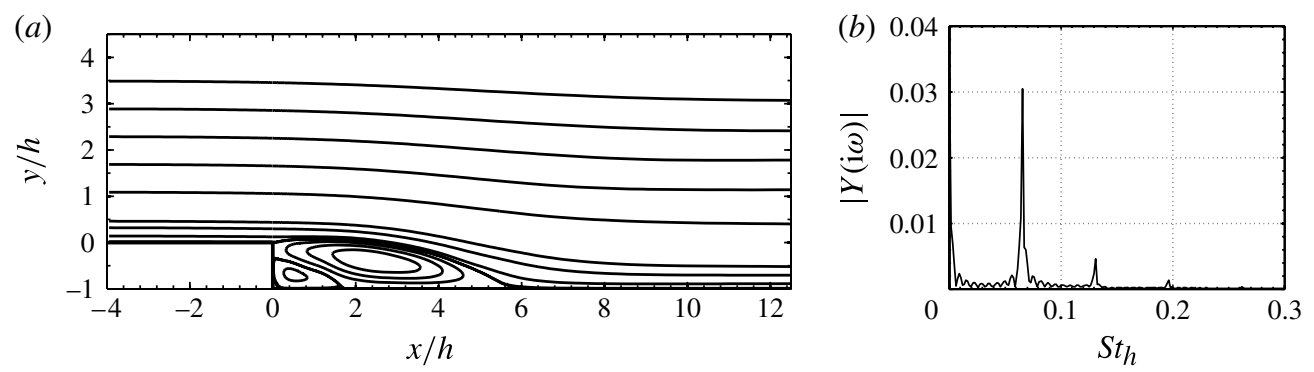

FIGURE 5. Unforced flow characteristics: (a) contours of time-averaged streamfunction; and (b) amplitude spectrum of base pressure coefficient (sensor signal).

describing functions methodology (Gelb \& Vander Velde 1968). Therefore, harmonic forcing is used in the present work for system identification. A linearity check is carried out by forcing at three different amplitude levels.

\section{Two-dimensional case}

In the first instance, a 2D planar domain is considered. Although the imposed 2D confinement of the flow prevents the development of turbulence that would arise at the present Reynolds number $\left(R e_{h}=2000\right)$ in a 3D domain, the results provide an initial evaluation of the feedback control strategy targeting the pressure fluctuations.

\subsection{Unforced flow}

The baseline flow is first examined to establish a reference against which to contrast the control results. Figure 5(a) shows the time-averaged streamlines for the unforced flow while figure $5(b)$ shows the spectrum of the base pressure coefficient. The shear layer emanating from the step edge grows and rolls up into a large-scale vortical structure under the combined influence of an adverse pressure gradient and presence of the lower wall. The mean reattachment point is observed at $x / h=6.1$, corresponding to the location of zero wall shear stress. The reattachment length is sensitive to the Reynolds number, the expansion ratio of the step and the state and thickness of the boundary layer at separation, with a low expansion ratio laminar flow expected to yield a reattachment length of around $6 h$ (Eaton \& Johnston 1981). A counter-rotating corner eddy extending up to $x / h=1.8$ is also captured in the time-averaged solution. This feature has been observed experimentally, confined between the step foot and $x / h=2$ (Driver \& Seegmiller 1985).

As the large coherent structure formed by the shear layer grows, the reattachment location travels downstream. An abrupt detachment of this structure leads to a reduction in the bubble size. This periodic phenomenon causes the separation bubble to oscillate around the mean reattachment point. The amplitude spectrum of the base pressure indicates that the flow near the step is dominated by the shear layer instability mode. The corresponding non-dimensional frequency is found to be approximately $S t_{h}=f h / U_{0}=0.0655$, or $S t_{\theta}=0.0092$, which agrees relatively well with the value reported by Hasan (1992), $S t_{\theta}=0.012$, for a backward-facing step with laminar separation. 

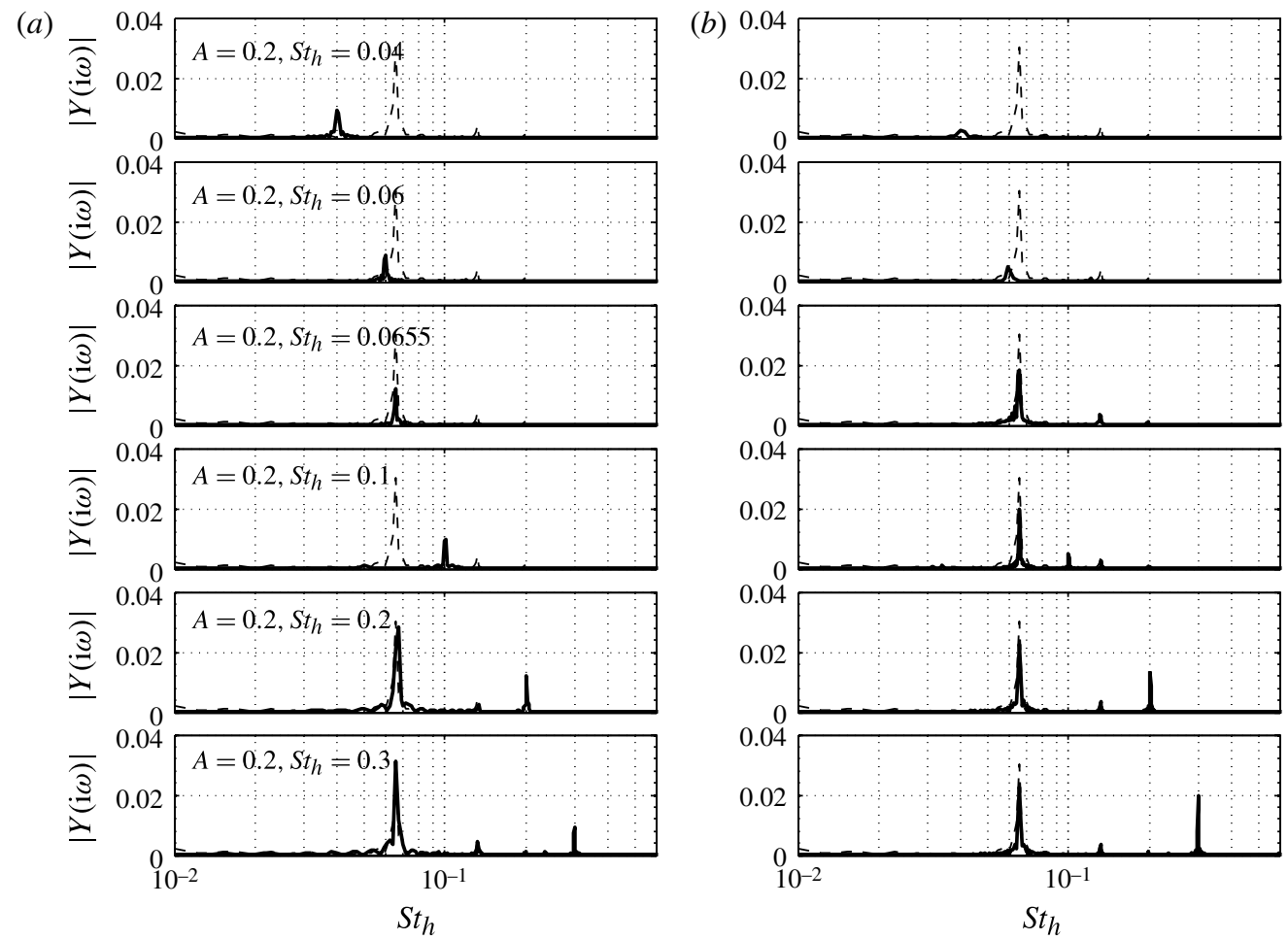

FIGURE 6. Amplitude spectra for (open-loop) harmonic forcing with (a) actuator 1 and (b) actuator 2 . The solid line is the forced signal and the dashed line is the baseline.

\subsection{Open-loop forcing and system identification}

After characterizing the unforced flow, the transfer function between the actuator signal and the sensor measurement, denoted by $G(s)$ in figure 4 , is to be determined using system identification. The flow is thus subjected to harmonic forcing via the slot jets described in $\S 2.3$. The forcing frequency is varied over the range $0.04 \leqslant S t_{h} \leqslant 1$ and three amplitudes are examined for each frequency $\left(A_{j} / U_{0}=0.1,0.2\right.$ and 0.3$)$. Actuators 1 and 2 are considered independently.

The base pressure amplitude spectra for various forcing frequencies at a fixed injection amplitude of $A_{j}=0.2$ are shown in figure 6. The flow is left to develop for 15 flow-through times after the start of the forcing to ensure the effects of transients are discarded. A first observation, common to both actuator locations, is that the flow response is strongly dependent upon the jet frequency. At frequencies near the dominant instability $S t_{h}=0.0655$, the shear layer locks in to the forcing, after transients. The time-averaged pressure $\overline{C_{P}}$ increases as a result of the frequency lockin, which is associated with a decrease in pressure fluctuations. In contrast, frequencies away from the lock-in region do not suppress the shear layer instability, for the range of amplitudes tested. Instead, beating is observed; both unforced and actuation frequencies are present in the response. As evidenced by figure 6 , the lock-in range is larger for actuator 1 , which acts directly onto the shear layer, whereas actuator 2 is buried within the wake. Interestingly, the response to actuator 2 appears to increase rapidly with frequency. 

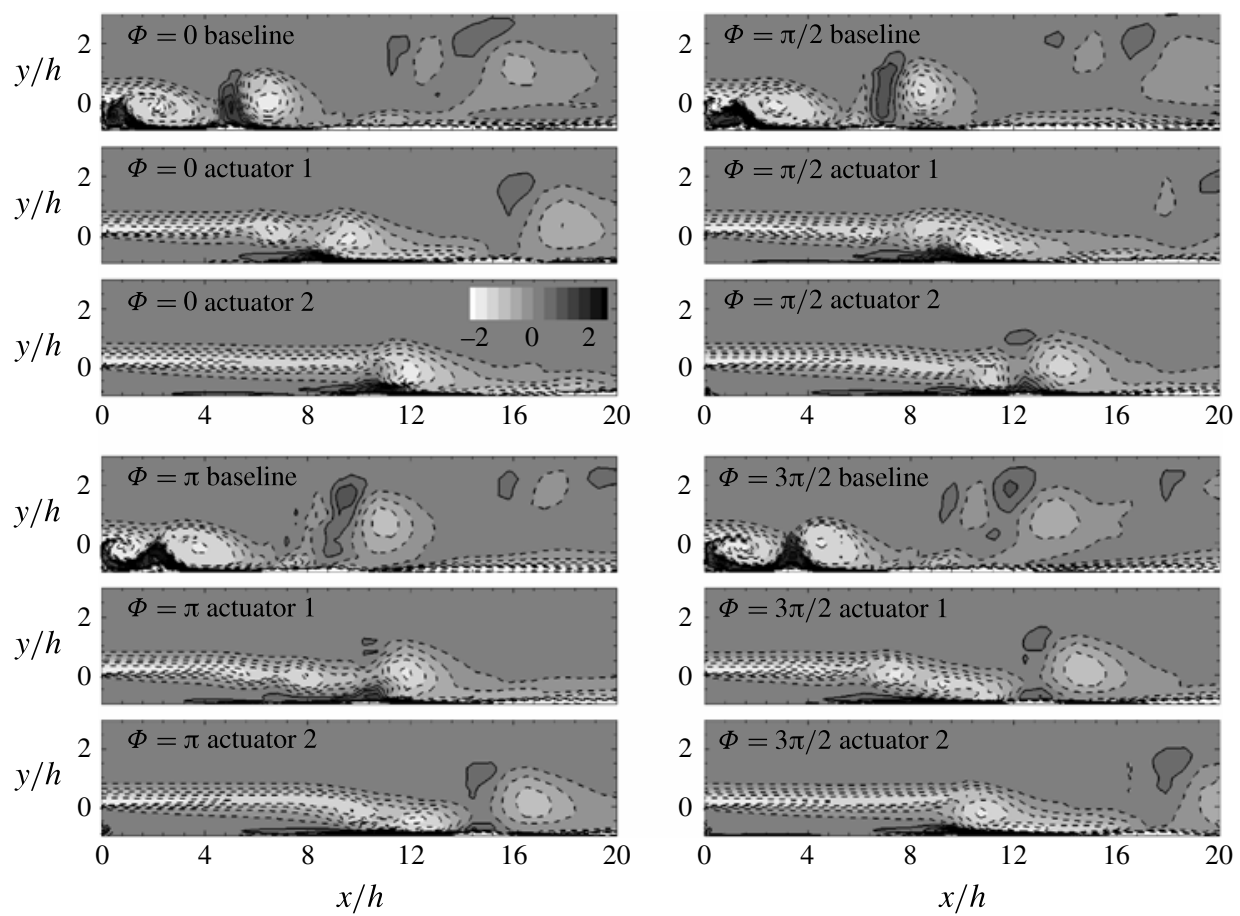

FIGURE 7. Phase-averaged vorticity contours (dashed lines for negative and solid lines for positive vorticity) for the baseline (averaging frequency $S t_{h}=0.0655$ ) and open-loop controlled $\left(A_{j}=0.2\right.$ and $\left.S t_{h}=0.04\right)$ flows for both actuators 1 and 2 downstream of separation.

The gain and phase shift of the response are found using spectral analysis, by expanding the base pressure signal into a Fourier series,

$$
C_{P}(t)=\sum_{n=0}^{N} a_{n} \sin \left(\omega_{n} t\right)+b_{n} \cos \left(\omega_{n} t\right),
$$

and evaluating the magnitude and the argument of the term corresponding to the forcing. Hence, the gain and phase shift of the response modes are recorded over the frequency range considered. Linearity is then confirmed by verifying that changing the input amplitude leaves the gain and phase shift - between the input and output signals - unchanged. In the present 2D study, the gains and phase shifts recorded with the three different amplitudes tested vary by less than $4 \mathrm{~dB}$ and $13^{\circ}$ respectively, for any given frequency within the considered range. These variations are small hence the response of the base pressure fluctuations to the forcing can be considered to be dynamically linear, within the range of parameters examined.

Information pertinent to the effect of the jet injection in its different stages may be derived from examining phase-averaged results. The unforced flow is also phaseaveraged at various phases of the dominant wake motion $\left(S t_{h}=0.0655\right)$. Figure 7 compares phase-averaged vorticity fields downstream of the step, for the unforced flow and that forced at $S t_{h}=0.04$, which leads to lock-in and base pressure increase. In the unforced case, the large vortical structures emerging from the shear layer rapidly roll up, convect downstream at a velocity of $0.4 U_{0}$ and detach abruptly in 

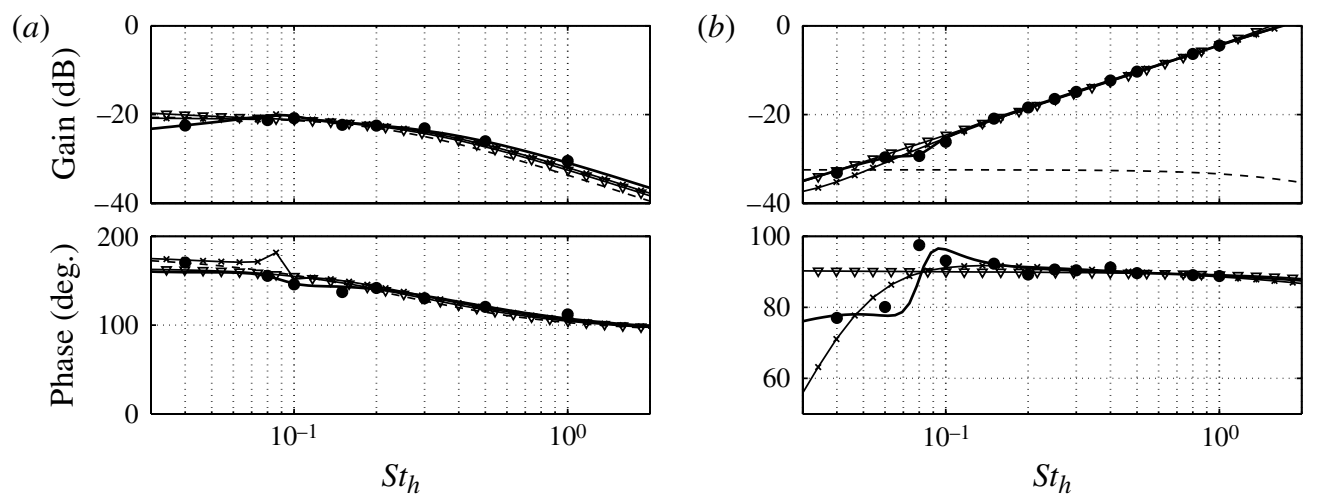

FIGURE 8. Frequency response $G(\mathrm{i} \omega)$ for $(a)$ actuator 1 , and $(b)$ actuator 2 : •, harmonic forcing; ----, fit order one; $\nabla$, fit order two; $\times$, fit order three; - , fit order four. The fits are obtained via least-squares minimization (using fitfrd) and the order refers to the denominator order of the resulting transfer function.

the reattachment region. After reattachment, the large-scale vortices lose coherence but remain visible for a long downstream distance. The low-frequency forcing significantly alters the shear layer dynamics with both actuators. It delays the roll-up and thereby elongates the recirculation bubble. This mechanism reduces the interaction of lowpressure structures, carrying high-momentum fluid, with the base wall. As a result, unsteadiness in the near-wake is reduced and the time-averaged pressure on the base increases. The shed structures also appear to be smaller with the forcing. Note that both actuators have a similar effect, although actuator 2 delays reattachment further.

The open-loop responses corresponding to both actuator locations, $G_{a c t u 1}(\mathrm{i} \omega)$ and $G_{a c t u 2}(\mathrm{i} \omega)$, are summarized in figure 8. The dots are gain and phase information obtained from the harmonic forcing and the lines show low-order models obtained by fitting four systems (of denominator order one, two, three and four) to the data via the 'fitfrd' MATLAB command. For the first actuator location the second-order fit provides a good match, while the fourth-order fit is chosen for the second location. The fit equations are as follows:

$$
\begin{gathered}
G_{a c t u 1}(s)=\frac{-0.154 s-0.0235}{s^{2}+1.776 s+0.168}, \\
G_{a c t u 22}(s)=\frac{-745.5 s^{3}-148.4 s^{2}-195.3 s-7.25}{s^{4}-27.89 s^{3}-7758 s^{2}-1705 s-2029} .
\end{gathered}
$$

For actuator 1, the transfer function is minimum phase (i.e. it has neither poles nor zeros with a positive real part) and has relatively flat dynamics, acting as a low-pass filter with a constant gain drop rate of $\sim 20 \mathrm{~dB}^{\text {decade }}{ }^{-1}$ at high frequencies. As already noted from figure 6 , the response to actuator 2 has a gain increasing with frequency. In addition, a phase increase is observed across the shear layer instability, corresponding to an unstable pole which will need to be stabilized by the controller.

\subsection{Feedback control}

In light of the measured open-loop responses, simple controllers are designed, targeting a reduction in the fluctuation levels. Based on figure 4, one can infer the 
(a)

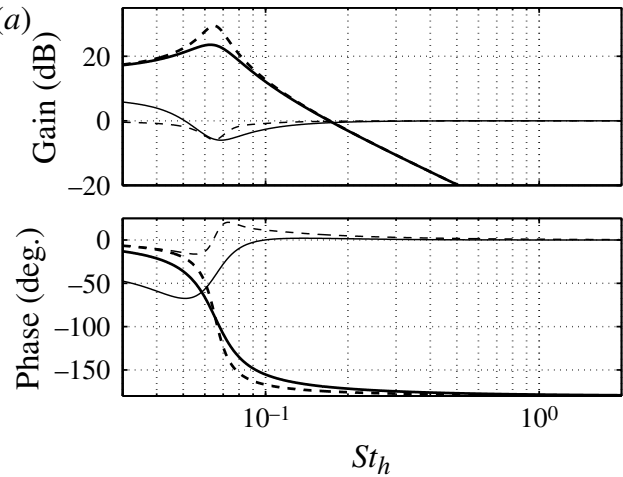

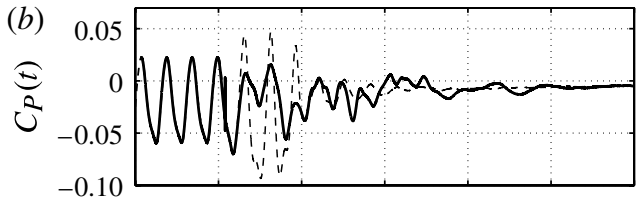

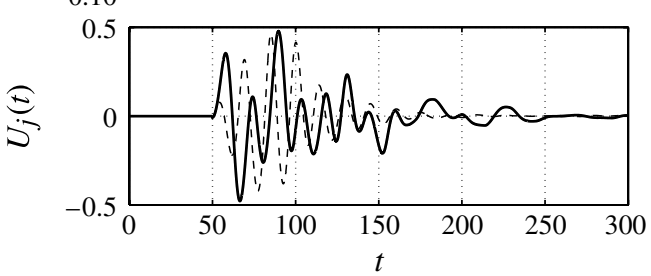

FIGURE 9. (a) Characteristics of closed-loop systems for both actuator locations. The gain and phase of the controllers $K$ are shown as thick lines whilst thin lines represent sensitivity function $S=1 /(1+G K)$. (b) Time variations of input $U_{j}(t)$ and output $C_{P}(t)$ (including mean component) for the controlled system. Solid and dashed lines refer to actuators 1 and 2 respectively. The controllers are turned on at $t=50$.

ratio of outputs $Y(s)$ with and without control, as follows:

$$
\frac{\left.Y(s)\right|_{\text {with control }}}{\left.Y(s)\right|_{\text {without control }}}=\frac{1}{1+G(s) K(s)}=S(s) .
$$

As the objective of the feedback control is to reduce the amplitude of base pressure fluctuations, it is clear that the ratio (3.4) needs to have magnitude less than unity. That is, a reduction in the output amplitude can be achieved via a controller $K(s)$ such that the denominator in (3.4) has magnitude larger than 1. This synthesis for fluctuation attenuation translates into a condition on the sensitivity function $S(s)$ to have a gain below unity over the range of frequencies at which the system operates. It is also necessary to ensure that the controller satisfies closed-loop stability.

A second-order polynomial controller $K(s)=1 /\left(s^{2}+2 \xi \omega_{n} s+\omega_{n}^{2}\right)$ is selected for both actuator locations and is implemented in the LES code. The resonant frequency is set to the dominant instability $\omega_{n}=2 \pi \times 0.0655$ and the damping coefficients $\xi=0.2$ (actuator 1) and $\xi=0.1$ (actuator 2) are chosen to ensure that the actuation signal does not deviate far off from the linear range of forcing amplitudes. Some of the characteristics of $K$ are shown in figure $9(a)$, where the sensitivity gain can be observed to be below unity around the shear layer instability frequency. Both actuator locations produce a similar response by stabilizing the near-wake and pushing the unsteady reattachment region further downstream, an effect akin to that observed in open-loop with the lock-in phenomenon. The stabilizing effect of the controller was expected since disturbance attenuation and stabilization are closely linked in control theory. After transients the base pressure oscillations are completely suppressed (figure $9 b$ ). This leads to a $70 \%$ increase in the time-averaged pressure. Thus, in these 2D BFS simulations, feedback control targeted at reducing fluctuations in the base pressure has indeed resulted in a form-drag reduction. In closed-loop, the work done by the actuator adapts to the evolution of the flow (here, for example, requiring only minimal input once the flow has been stabilized), which is a distinct advantage over open-loop. 

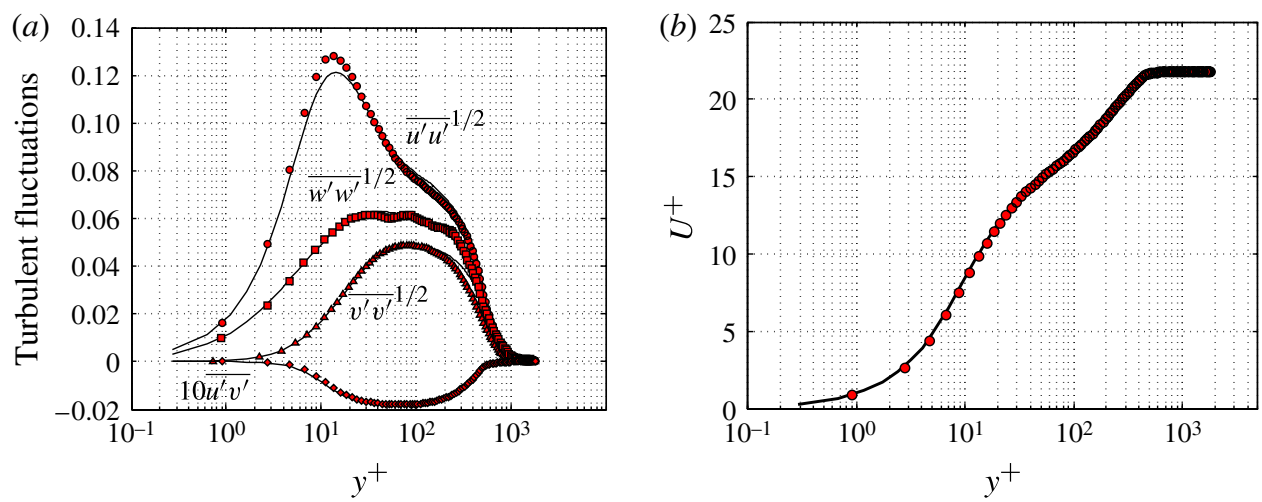

FIGURE 10. (Colour online) Flow characteristics at separation $(x / h=0)$ compared with DNS data from Jimenez et al. (2010) (solid lines) for a fully developed zero-pressure gradient boundary layer. (a) The root-mean-square turbulent fluctuations and the Reynolds stress. (b) Plot of the mean streamwise velocity profile.

\section{Three-dimensional case}

Attention now turns to the more physically representative 3D BFS flow with a turbulent boundary layer at separation. The domain illustrated in figure 1 is considered, with $R_{\theta}=1500$. A similar procedure as for the $2 \mathrm{D}$ flow is followed but with more emphasis on the effects of the forcing on the flow. The unforced flow solution is first examined, after which system identification is carried out and controllers are synthesized for both actuator locations.

\subsection{Unforced flow}

Figure 10 shows the turbulent second moments and mean streamwise velocity profile at separation, compared with the DNS data of Jimenez et al. (2010) for a turbulent boundary layer at $R e_{\theta}=1551$, with zero streamwise pressure gradient. Good agreement is obtained, confirming a satisfactory representation of the incoming turbulent boundary layer in the LES simulations. In figure 11, time-averaged velocity and Reynolds stress profiles in the initial stages of the separated shear layer are compared with experimental data, obtained by particle image velocimetry (PIV) on a BFS flow at $\operatorname{Re}_{\theta}=1381$ (Brosco 2011). Despite some small discrepancies in the streamwise and transverse normal stresses, the overall conclusion is that the LES is capturing the unforced flow well.

In the following, we investigate whether the spanwise extent $L_{z}=4 h$ is sufficient. Structures with large scales in the spanwise direction may exist in spanwisehomogeneous flows and some error is induced if the domain does not contain these fully. The two-point spanwise correlation coefficient $R$ gives an indication of the spanwise extent of those structures. The definition of the correlation coefficient used here is

$$
R_{\phi \psi}(\Delta z)=\frac{\overline{\phi(z) \psi(z+\Delta z)}}{(\overline{\phi(z) \phi(z)} \overline{\psi(z+\Delta z) \psi(z+\Delta z)})^{1 / 2}},
$$

where $\phi$ and $\psi$ are two fluctuating quantities. Figure 12 displays the correlation coefficients $R_{u u}$ and $R_{p p}$ along three spanwise lines inside the shear layer. For both the streamwise velocity and the pressure, the correlation levels decrease rapidly and 

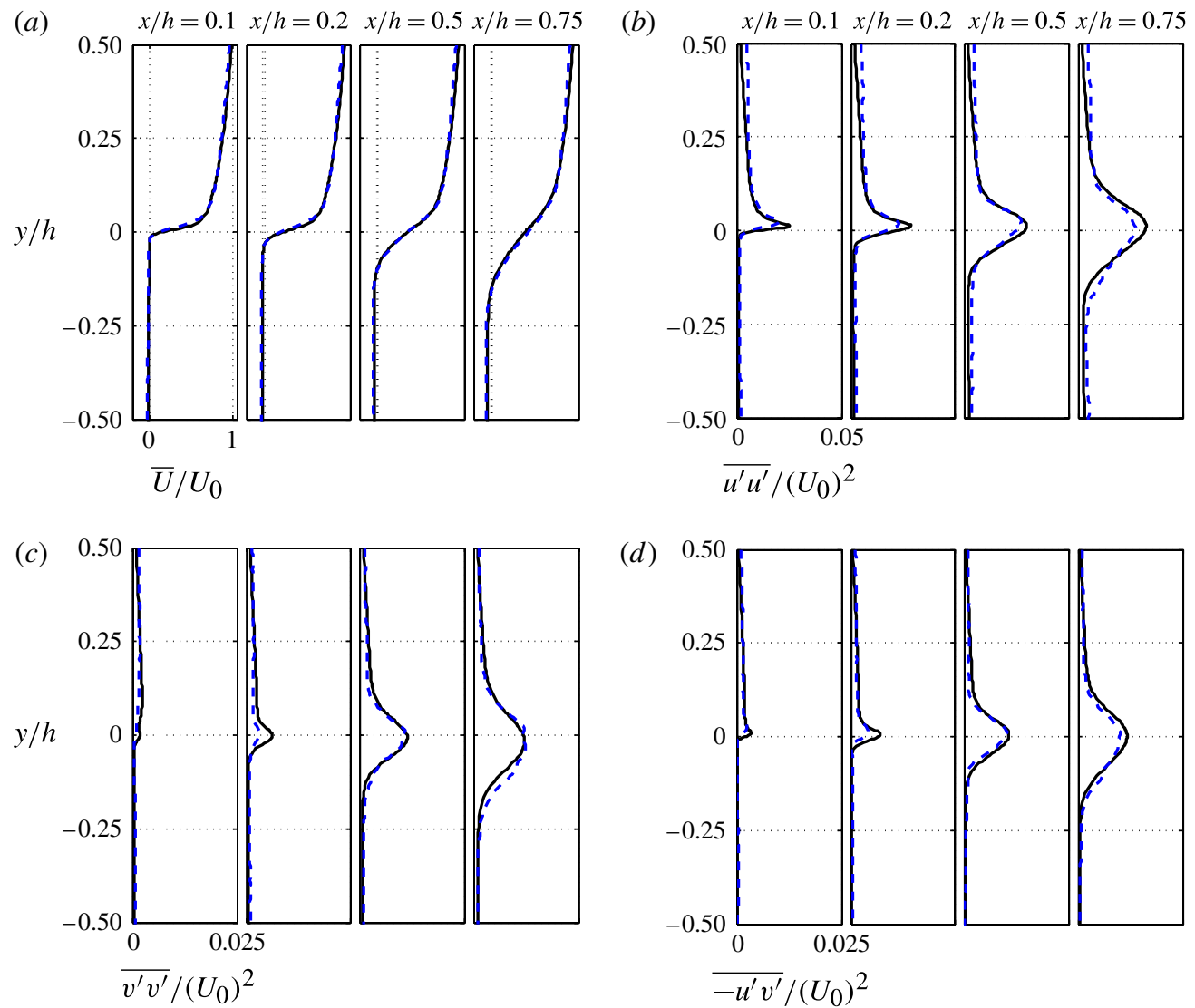

FIGURE 11. (Colour online) Time-averaged velocity and Reynolds stress profiles compared via LES (solid lines) and PIV from Brosco (2011) (dashed lines). (a) Mean streamwise velocity; $(b)$ streamwise normal stress; $(c)$ transverse normal stress; $(d)$ Reynolds shear stress.

remain close to zero for $\Delta z / h>0.5$. Note that the correlation does not vanish fully within a half-domain width $(2 h)$, which suggests that a larger spanwise extent may improve the accuracy of the results. Nevertheless, the spanwise extent $L_{z}=4 h$ is deemed acceptable.

The unforced base pressure spectrum is shown in figure 13(a). A wide range of scales, characteristic of turbulent flows, is observed. A peak appears around $S t_{h}=0.32$ which corresponds to $S t_{\theta}=0.0176$. This peak is associated with the convective shear layer instability. Indeed, it is also visible in the initial stage of the separating shear layer where this instability originates (see figure $13 b$ at $x / h=1$ ). According to Hasan $\&$ Khan (1992), the shear layer instability is characterized by $S t_{\theta}=0.011$ for a BFS flow with a turbulent separation. However, there are varying opinions on this in the literature and the instability frequency decreases as the shear layer develops. Pressure spectra at two downstream locations $(x / h=1, y / h=0)$ and $(x / h=6, y / h=-0.25)$ are presented in figure $13(b)$. The shear layer instability $\left(S t_{\theta}=0.0176\right)$ appears near separation and the shedding mode $\left(S t_{h} \approx 0.25\right)$ is observed further downstream, near reattachment. The reattachment length is $X_{r_{0}}=6.2 \mathrm{~h}$ for the baseline case. With the fine grid used for validation $X_{r 0}=6.5 h$ is obtained. The difference of $4.8 \%$ is small given 


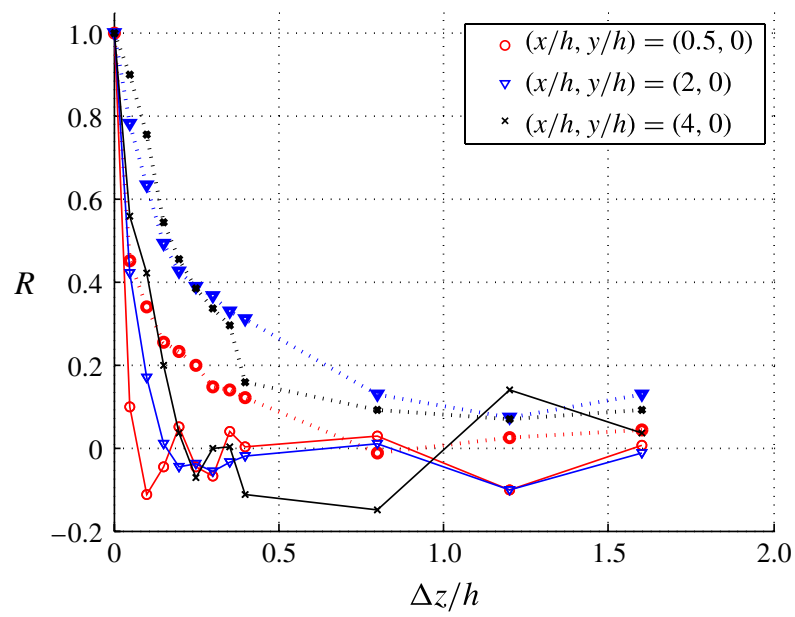

FIGURE 12. (Colour online) Correlation coefficients $R_{u u}$ (solid lines) and $R_{p p}$ (dotted lines) for varying spanwise distance $\Delta z$ in the separated shear layer.
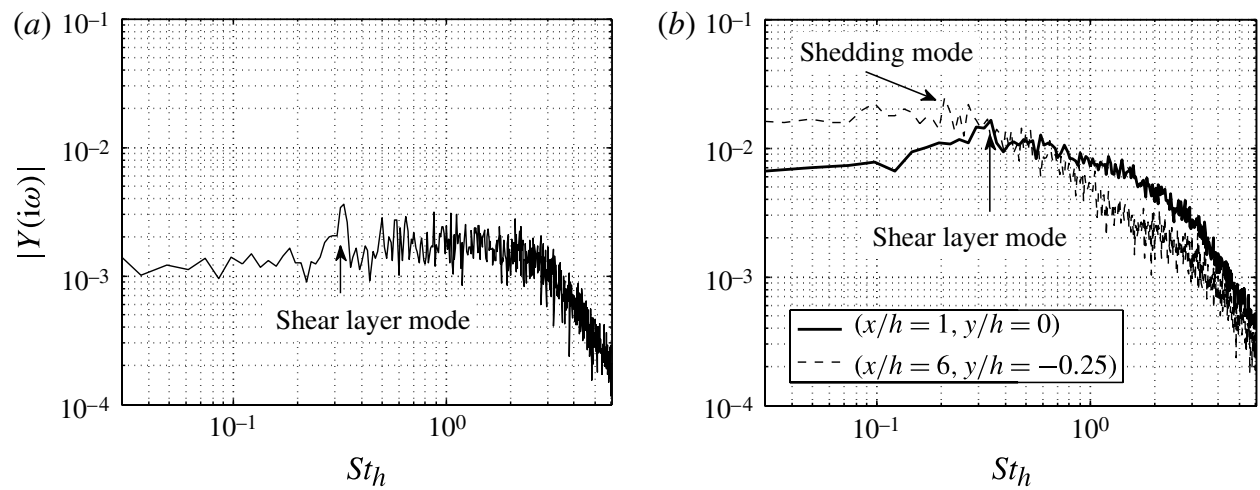

FIgURE 13. (a) Amplitude spectrum of sensor signal (spatially averaged base pressure fluctuations) for the baseline flow. (b) Pressure amplitude spectra at $(x / h=1, y / h=0)$ and $(x / h=6, y / h=-0.25)$ (signals averaged along the $z$ direction).

that the reattachment length is very sensitive to flow conditions upstream of separation and that the boundary layers developing along the inlet plate are likely to develop slightly different features with different grids. In addition, the value of $6.2 \mathrm{~h}$ agrees qualitatively with the guidelines given in Eaton \& Johnston (1981) and with the value of $6.28 h$ obtained by Le, Moin \& Kim (1997).

\subsection{Open-loop forcing and system identification}

After investigating the baseline flow, the purpose of this section is two-fold. Firstly, the effects of the open-loop forcing (with both actuator locations) on the flow are described. Secondly, the results of the system identification procedure carried out in a similar manner to $\$ 3.2$ are discussed.

Figure 14 illustrates the effect of the slot jet forcing frequency on two time-averaged quantities: the reattachment length $X_{r}$ and the (spatially averaged) base pressure $\overline{C_{P}}$. 

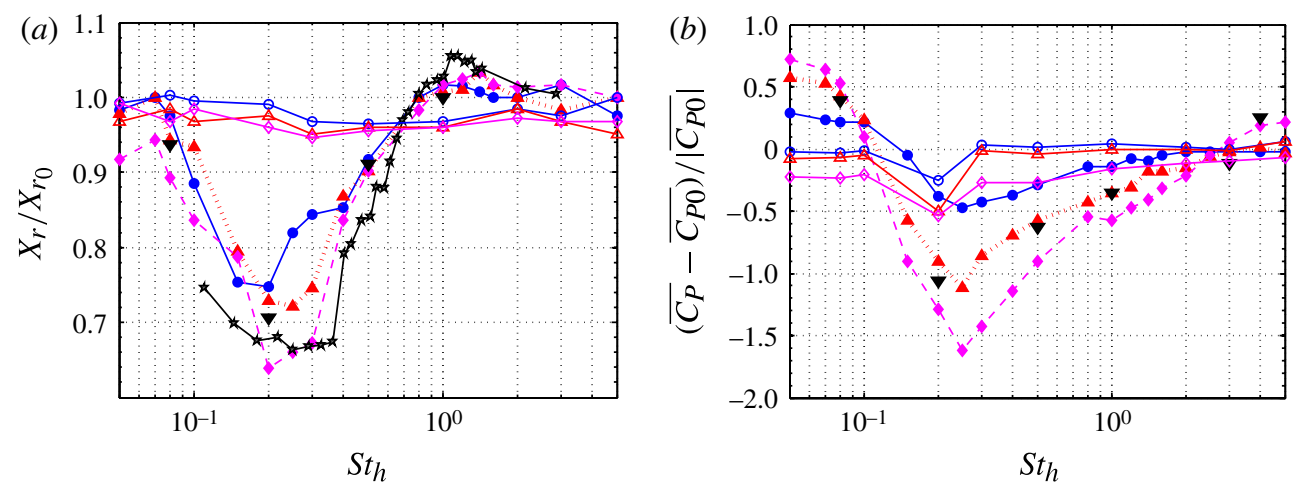

FIGURE 14. (Colour online) (a) Effect of open-loop forcing on time-averaged reattachment length $X_{r}$ ( $X_{r 0}$ is baseline reattachment length). Experimental data from Chun \& Sung (1996) $\left(R e_{\theta}=890\right.$, forcing level $\left.A_{0}=0.07\right)$ is included for comparison ( $\left.\star\right)$. (b) Change in time-averaged base pressure $\overline{C_{P}}\left(\overline{C_{P 0}}\right.$ is baseline pressure). Three actuation amplitudes are considered: $\bullet, A_{j}=0.1 ; \Delta, A_{j}=0.2 ; \diamond, A_{j}=0.3$. Results obtained at a few frequencies with actuator $1\left(A_{j}=0.2\right)$ on the fine grid are denoted by $\mathbf{\nabla}$. Filled symbols correspond to actuator 1 and open symbols to actuator 2 .

The most evident feature is the radically different response of the wake to the two actuator locations. Actuator 1, located near the separation edge in a region of high receptivity, has a strong impact on both $X_{r}$ and $\overline{C_{P}}$. On the contrary, actuator 2, perturbing the flow near the base foot, has virtually no impact on the reattachment length. It leads to a reduction in $\overline{C_{P}}$ with a narrow valley at $S t_{h} \approx 0.25$ and a roughly constant pressure decrease (which strengthens with the amplitude of the perturbation) away from this frequency.

For actuator 1, figure 14(a) shows that forcing decreases $X_{r}$ by up to $35 \%$ at low forcing frequencies and increases it slightly for frequencies in the range $0.8 \leqslant S t_{h} \leqslant 2$. A comparison with experimental data at $R_{\theta}=890$ from Chun \& Sung (1996) is included. Although the flow conditions and actuation level differ (the latter cannot be directly compared due to different slot widths and definitions of the forcing amplitude), the curve for forcing level $A_{j}=0.3$ agrees reasonably well with the experimental data. Yoshioka, Obi \& Masuda (2001) report a maximum reduction in reattachment length of $30 \%$ for a forcing amplitude $A_{j}=0.3$, which provides further support for the present results. The reduction of the reattachment length is linked to increased turbulent stresses near separation, which lead to a higher growth rate of the shear layer. The shear near the interface of the layer and the recirculating flow is also higher, thus producing higher entrainment and earlier reattachment.

We now turn our attention to the effect of actuator 1 on the time-averaged base pressure $\overline{C_{P}}$. Figure $14(b)$ shows that frequencies below $S t_{h} \approx 0.1$ lead to an increase in $\overline{C_{P}}$ whereas a pressure decrease is observed for $S t_{h}>0.1$. The change in $\overline{C_{P}}$ is nearly a linear function of forcing amplitude within the frequency range $0.1<S t_{h}<2$ for the three amplitudes tested, although the relation becomes nonlinear if higher forcing amplitudes are used.

For both the reattachment length and the base pressure coefficient, results obtained with the fine grid at a few selected forcing frequencies match well with the solutions on the coarser grid, thereby indicating that the latter grid is adequate to compute the perturbed flows. However, a significant discrepancy is observed in the time-averaged 
(a)

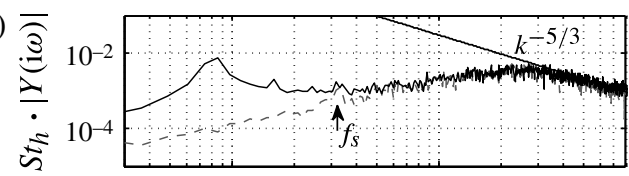

(b)

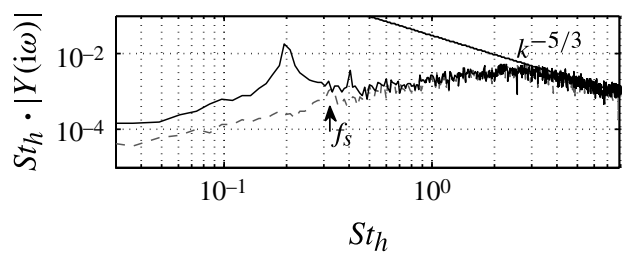

(c)

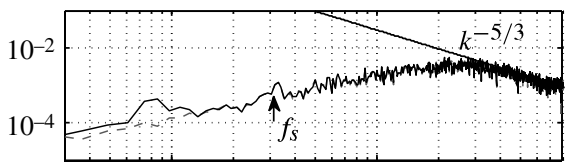

$(d)$

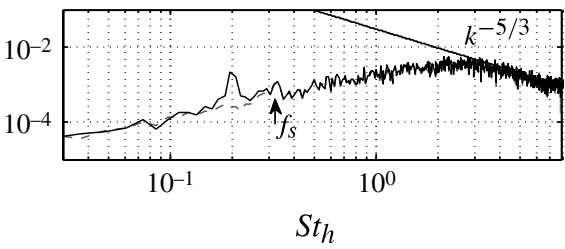

FIGURE 15. Spectra of spatially averaged base pressure (sensor signal) for harmonic forcing at $(a, c) S t_{h}=0.08$, and $(b, d) S t_{h}=0.2$. $(a, b)$ Actuator $1,(c, d)$ actuator 2. The convective shear layer instability present in the baseline flow is denoted by $f_{s}$.

base pressure obtained for $S t_{h}=4$, as evidenced by figure 14(b). The coarse grid predicts that $\overline{C_{P}}$ is not affected by the harmonic perturbations with $A_{j}=0.2$ for $S t_{h}>3$ whilst the fine grid shows a pressure increase of almost $30 \%$ for $S t_{h}=4$. This means that the coarse grid is not able to fully resolve the small-scale structures produced by high-frequency perturbations (an order of magnitude above the dominant unstable modes of the flow). Interestingly, one might hence suggest that actuation via these small-scale perturbations provides a promising mechanism for base pressure increase.

For all three forcing amplitudes, global minima are obtained for both $X_{r}$ and $\overline{C_{P}}$ around the same frequency $S t_{h} \approx 0.25$. A number of studies on reattaching shear layers have proposed that the frequency for a maximum reduction of $X_{r}$ is associated with the shedding mode (see e.g. Dandois, Garnier \& Sagaut 2007). In other words, the frequency for maximum reduction of $X_{r}$ and $\overline{C_{P}}$ is dictated by the rate of vortex shedding from the reattachment region. As shown in figure 14(a), Chun \& Sung (1996) obtained a global minimum at $S t_{h} \approx 0.27$, and they associated this extremum with the shedding mode. Also, they observed the existence of a local minimum at $S t_{h}=0.4$ for lower forcing amplitudes, which they identify as the shear layer instability. A pronounced dip is visible in figure $14(a)$ at $S t_{h}=0.4$ for the lowest amplitude $A_{j}=0.1$. This supports the hypothesis that the forcing frequency $S t_{h} \approx 0.25$ corresponding to the trough in the $X_{r}$ and $\overline{C_{P}}$ bucket is in fact linked to the shedding mode.

Figure 15 illustrates base pressure spectra for the flows perturbed with two open-loop harmonic signals $U_{j}=0.2 \sin (2 \pi 0.08 t)$ and $U_{j}=0.2 \sin (2 \pi 0.2 t)$. We will henceforth term those two signals the low-frequency (LF) and the medium-frequency (MF) forcing. Both actuators are considered and compared to the baseline case. Comparison of the two actuators leads to an observation already mentioned: open-loop control with actuator 1 has a stronger impact on the flow. Large peaks associated with the forcing are visible for actuator 2 for both the LF and MF perturbations. These peaks, however, are smaller than those produced by actuator 1 and other wavelengths are not sensibly disturbed compared to the baseline flow. Actuator 1 amplifies the level of pressure fluctuations on the base over a broad range of scales around the forcing frequency. In addition, it can be observed that the MF control with actuator 1 tends to suppress the shear layer instability. 

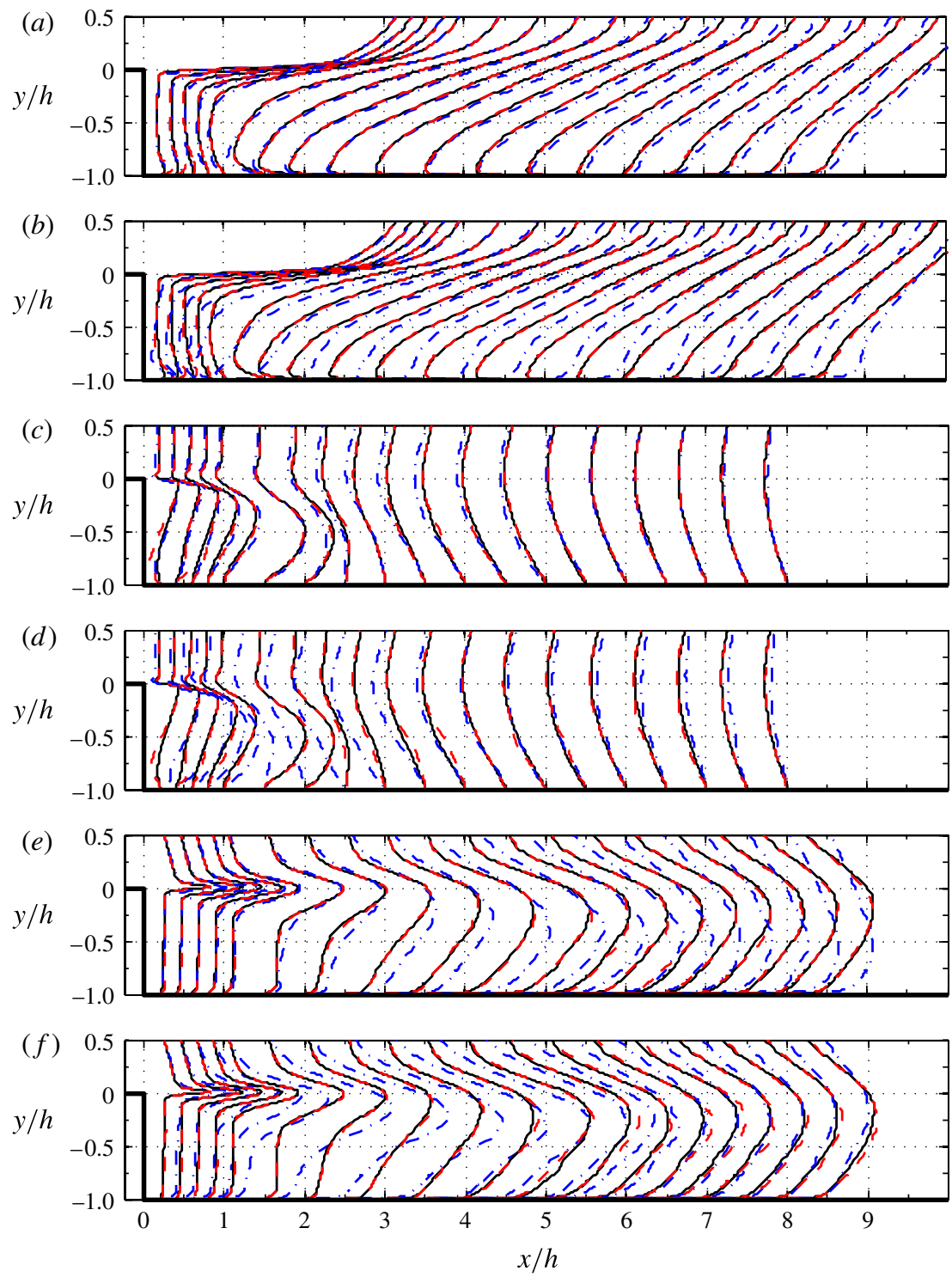

FIGURE 16. (Colour online) Flow characteristics downstream of separation for forcing with $(a, c, e) S t_{h}=0.08$, and $(b, d, f) S t_{h}=0.2$ for $A_{j}=0.2$. $(a, b)$ Time-averaged streamwise velocity profiles $\bar{U}$. $(c, d)$ Time-averaged transverse velocity $\bar{V}$. $(e, f)$ Turbulent kinetic energy $k=0.5\left(\overline{u^{\prime} u^{\prime}}+\overline{v^{\prime} v^{\prime}}+\overline{w^{\prime} w^{\prime}}\right)$. Lines: — , unforced flow; -...., actuator 1 ; - - - - , actuator 2 . The magnitudes of the profiles have been scaled for clarity.

Figure 16 shows some features of the flow perturbed with the LF and MF signals. Again it is obvious that the second actuator has a lesser impact on statistical flow features compared to the first one. However, careful observation of profiles of $\bar{U}$ and $\bar{V}$ in figure 16 reveals that the mean flow velocity profiles in the near-separation region are altered by actuator 2 . Considering actuator 1 , it was previously observed that the $\mathrm{LF}$ forcing increases the mean base pressure $\overline{C_{P}}$, whilst the MF forcing reduces it. As 
(a)

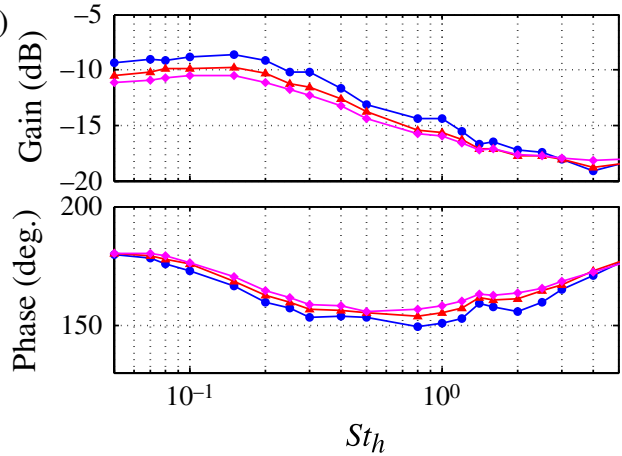

(b)
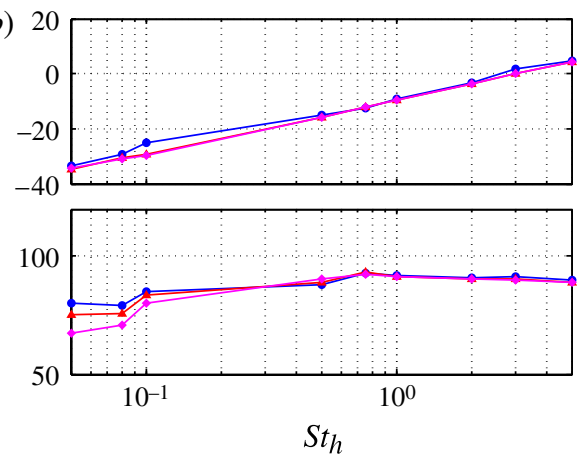

FIgURE 17. (Colour online) Bode plots of frequency responses $G(\mathrm{i} \omega)$ obtained using harmonic inputs with three forcing amplitudes: $\bullet, A_{j}=0.1 ; \Delta, A_{j}=0.2 ; \diamond, A_{j}=0.3$. (a) Actuator 1, (b) actuator 2 .

evidenced by figure $16(a, c)$, the mean velocity profiles are only weakly affected by the LF forcing. On the other hand, the MF forcing reduces the reattachment length more significantly and hence affects the velocity field. The turbulent kinetic energy profiles shown in figure 16(e) reveal an interesting perspective: the LF actuation dramatically changes the profile of turbulent fluctuations downstream of reattachment, where the influence of the MF forcing is comparatively small. The turbulent kinetic energy is pulled towards the lower wall by the LF forcing. As is well known, the forcing at $S t_{h}=0.2$ increases the peak turbulent fluctuations in the separated region, with a maximum reached around $x / h=2$.

As for the 2D flow, the information obtained from the harmonic open-loop forcing also serves to identify the flow response $G(\mathrm{i} \omega)$. The validity of the dynamic linearity assumption is checked by examining figure 17 , which shows the frequency responses $G_{1}, G_{2}$ and $G_{3}$ obtained with forcing at three different amplitudes $A_{j}=0.1,0.2$ and 0.3 , with each actuator independently. For both actuators, the slight mismatch between the three curves reveals that weak nonlinearities are present in both gain and phase. However, approximating the flow response as a dynamically linear process is a reasonable approximation here, as long as the magnitude of the actuator signal remains within the range considered.

For each actuator, a frequency response model $G$ is constructed as the average of $G_{1}, G_{2}$ and $G_{3}$, illustrated by the square dots in figure 18 . The solid line represents a model fitted to the data. The models fitted for actuators 1 and 2 are given by

$$
\begin{gathered}
G_{a c t u 1}(s)=\frac{-1440 s-9312}{s^{2}+11740 s+28560}, \\
G_{\text {actu2 } 2}(s)=\frac{62.88 s^{2}+1404 s+98.85}{s^{2}+1148 s+26427} .
\end{gathered}
$$

Both fitted transfer functions $G_{a c t u 1}$ and $G_{a c t u 2}$ are stable and minimum phase. The dashed line in figure 18 is a model obtained by performing the eigensystem realization algorithm (ERA) on input-output data from the LES. The input selected for the ERA is a sum-of-sines signal of the form (4.4). The lower and upper limits of the passband are $\omega_{\min }=0.3$ and $\omega_{\max }=31, K=24$ is the number of sinusoids spread evenly within the passband, and $\omega_{k}=\omega_{\min }+(k-1)\left(\omega_{\max }-\omega_{\min }\right) /(K-1), k=1, \ldots, K$. To ensure a sensible time variation of the signal amplitude, the phase of the $k$ th sinusoid at $t=0$ 
(a)

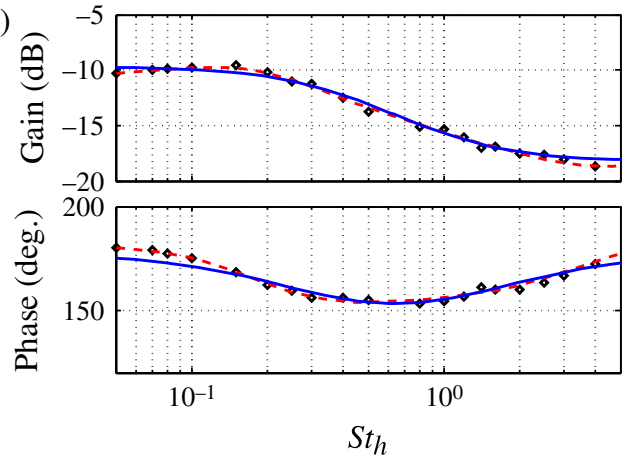

(b)

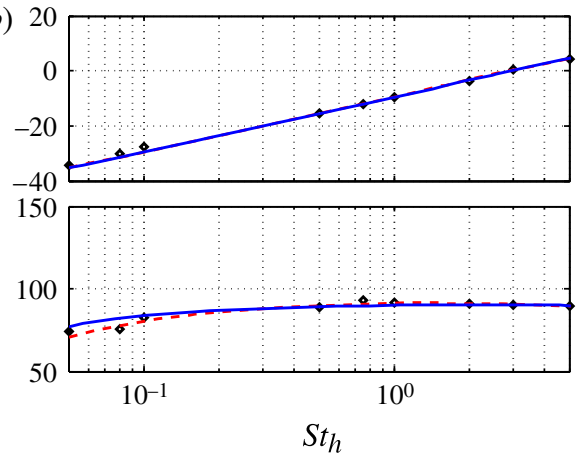

FIGURE 18. (Colour online) Bode plots of frequency responses $G(\mathrm{i} \omega)$ for $(a)$ actuator 1 , and (b) actuator 2 . The average over the three amplitudes shown in figure 17 is denoted by $\diamond$. The solid line denotes second-order models fitted to the data via fitfrd. The dashed lines represent models obtained via the eigensystem realization algorithm with a sum-of-sines input.

is $2 \pi \phi_{k}$, where $\phi_{k}$ is a random number taken from a uniform distribution on the interval $[0,1]$ :

$$
U_{j}(t)=A_{j} \sum_{k=1}^{K} \sin \left(\omega_{k} t+2 \pi \phi_{k}\right) .
$$

The ERA is a system identification and model reduction technique proposed by Juang \& Pappa (1985) which generates reduced-order models theoretically identical to those obtained from balanced POD (Ma, Ahuja \& Rowley 2009). The balancing refers to the observability and controllability Gramians of the reduced model being equal and diagonal (balanced), which ensures that the dynamics of the system are properly accounted for (by selecting the modes of the system which are both observable and controllable to reduce the system to a low-order model). An important feature of the ERA is that it does not require the solution of an adjoint system and hence is suitable for use with both computational and experimental data. For more details on the notions of observability and controllability and on the ERA, see Illingworth (2009). Figure 18 demonstrates good agreement in the transfer functions obtained by spectral analysis with harmonic forcing and those obtained with the ERA using a more sophisticated input signal. We conclude that both methods are suitable. The ERA is less computationally expensive but care must be taken to design an input signal with smooth time variations so as to avoid numerical instabilities.

Although the dynamics of $G$ here are different than in the 2D laminar case, some similarities are evident from comparison of figures 8 and 18. In particular, actuator 2 exhibits a gain increasing monotonically with frequency, within the frequency band examined, and a constant phase lag at high frequencies. On the other hand, the transfer function from the first actuator to the base pressure is highly sensitive to the shear layer development and hence has different dynamics in the laminar and turbulent flows. Indeed, the low-frequency gain is higher and there is no high-frequency roll-off in 3D.

It is important to keep in mind some of the limitations of the low-order models built herein; in particular that they apply only specifically to the actuation-sensing couple selected. As observed above, if the actuator is displaced to a different location, its impact on the wake is altered and different dynamics arise. 


\subsection{Feedback control}

After obtaining a low-order model for $G(s)$, a controller $K(s)$ can be designed using the fluctuation attenuation approach described in $\$ 3.3$. Once again, the controller aims to reduce the fluctuations in the sensor signal, by constraining the sensitivity function to have a gain below unity within a specific frequency range. Achieving this over a large frequency band is not always possible. Bode's integral formula (4.5) relates the integral of the sensitivity gain over all frequencies to the unstable open-loop poles $p_{k}$ :

$$
\int_{0}^{\infty} \ln |S(\mathrm{i} \omega)| \mathrm{d} \omega=\pi \sum \operatorname{Re}\left(p_{k}\right)-\frac{\pi}{2} \lim _{s \rightarrow \infty} s L(s) .
$$

This means that if the sensitivity gain is reduced in a particular frequency range, it will increase in another. Whilst this was of little concern in the 2D case, where the baseline flow is dominated by a single wavelength, the $3 \mathrm{D}$ turbulent flow requires the shaping of $|S(\mathrm{i} \omega)|$ over a large range of scales. Therefore, the $H_{\infty}$ loop-shaping method is chosen here (McFarlane \& Glover 1989). The loop-shaping technique, used in 2D, is a well-known approach in control theory, whereby one specifies closed-loop objectives in terms of requirements on the open-loop transfer function $L$ (or its singular values in the case of multi-input/multi-output systems). This approach however suffers from the need to ensure stability of the closed-loop system. Another approach to controller design is $H_{\infty}$ synthesis in which the closed-loop objectives are expressed in terms of weighted closed-loop transfer functions. $H_{\infty}$ synthesis guarantees stability and robustness, although the selection of the closed-loop weights is not straightforward and may be disconnected from the properties of the controlled process. $H_{\infty}$ loopshaping combines the advantages of these two methods. The open-loop properties of $L$ are specified first by adding a pre- and post-compensator to the process $G$, and the $H_{\infty}$ method is then used to robustly stabilize this shaped plant. Hence, this method returns a robust controller $K(s)$ to satisfy a desired open-loop transfer function $L(s)=G(s) K(s)$ whilst the closed-loop system is guaranteed to be stable. The characteristics of the resulting controllers for both actuator locations are as follows:

$$
\begin{gathered}
K_{1}(s)=\frac{-1.26 \times 10^{8}}{s^{4}+196 s^{3}+16660 s^{2}+504000 s+9 \times 10^{6}}, \\
K_{2}(s)=\frac{3000}{s^{2}+20 s+100} .
\end{gathered}
$$

Figure 19 shows the frequency responses of the controllers and the corresponding sensitivity functions $S(\mathrm{i} \omega)$ obtained from the control synthesis. The main features to note are that the first actuator location has lower stability margins, as well as a small positive hump in the sensitivity gain centred around $S t_{h}=7$ which implies that noise is amplified near this frequency.

Figure 20 illustrates the results obtained from implementing the feedback controller $K_{1}$ into the LES. The pressure fluctuations are reduced in amplitude and the actuation levels required are relatively low. The amplitude spectrum of the sensor signal given in figure 20(c) highlights an attenuation of the fluctuations over a large bandwidth, although high frequencies are amplified. The mean base pressure is also increased by $20 \%$ compared to the baseline flow. The control results for the controller $K_{2}$, operating via the second actuator location, are shown in figure 21. In this case, the attenuation of the pressure fluctuations and the high-frequency rejection are more effective than with $K_{1}$. However, very low frequencies are amplified, as evidenced by figure $21(c)$. The actuation signal $U_{j}$ output by the controller $K_{2}$ operates at low frequencies, in contrast 

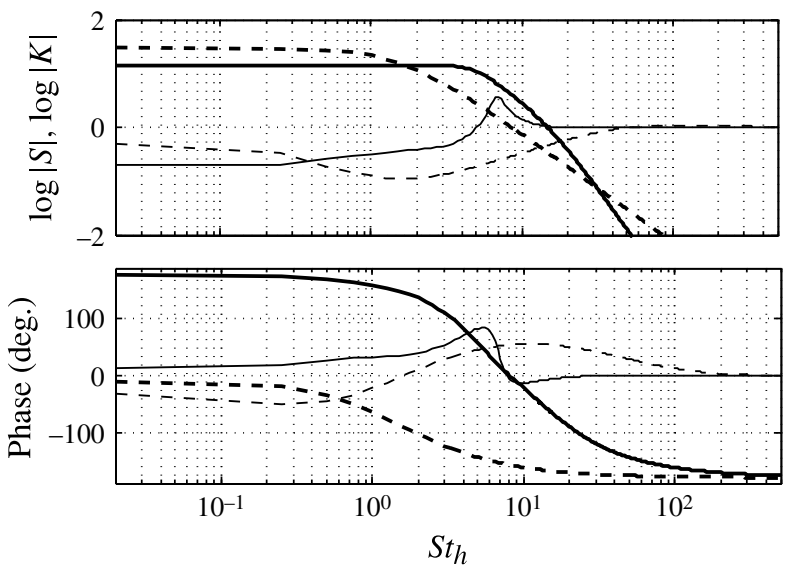

FIGURE 19. Characteristics of closed-loop systems for both actuator locations. Solid and dashed lines refer to the first and second actuators respectively. The gain and phase of the controllers $K$ are shown as thick lines whilst thin lines represent the sensitivity functions.

(a)

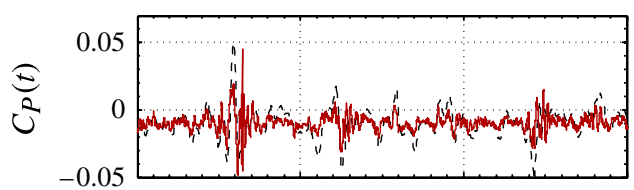

(b)

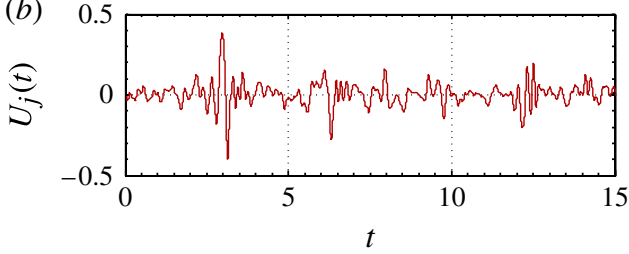

(c)
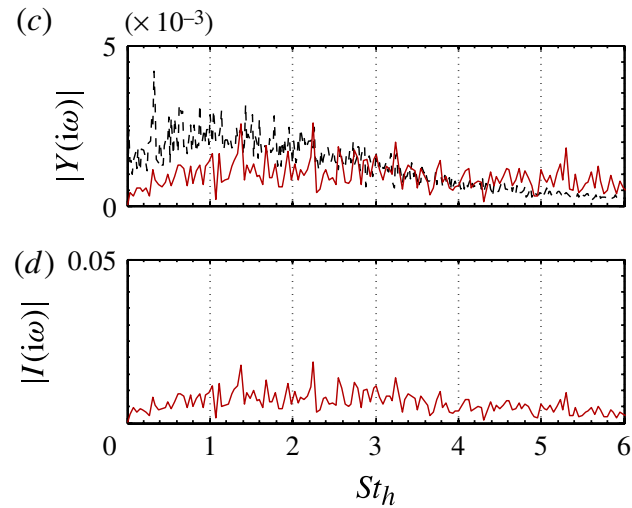

FIGURE 20. (Colour online) Input and output for controller $K_{1}$, with the first actuator location. Time-series of $(a)$ the base pressure signal and $(b)$ the actuation signal. Amplitude spectrum of $(c)$ the sensor signal and $(d)$ the actuation signal. Lines: ---- baseline; control.

to the previous controller. A mean base pressure increase of $10 \%$ is recorded with $K_{2}$, which is less than with $K_{1}$. This suggests that the link between the base pressure fluctuations and the time-averaged value is complex and further investigation into this relationship would need to be performed in order to exploit it for optimization.

Figure 22 shows some characteristics of the controlled flows downstream of separation compared to the baseline flow. The controllers $K_{1}$ and $K_{2}$ only mildly affect the time-averaged velocity fields $\bar{U}$ and $\bar{V}$. Nevertheless, the turbulent kinetic energy $k$ is significantly reduced over a large region of the domain. Actuator 2 leads to a more important reduction of $k$ in the separated shear layer.

Line contours of the time-averaged streamfunction for the baseline and controlled flows are plotted in figure 23. As discussed above, the time-averaged streamwise velocity field is globally only weakly modified by the feedback controllers for 
(a)

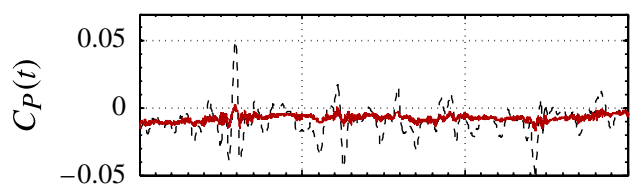

(b)

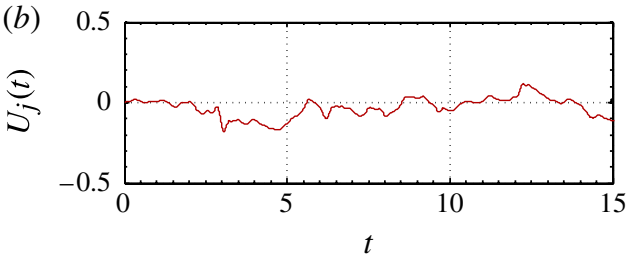

(c)
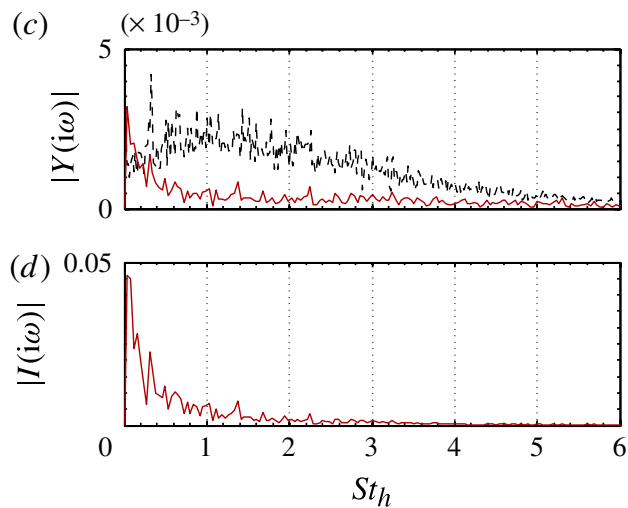

FIgURE 21. (Colour online) Input and output for controller $K_{2}$, with the second actuator location. Time-series of $(a)$ the base pressure signal and $(b)$ the actuation signal. Amplitude spectrum of $(c)$ the sensor signal and $(d)$ the actuation signal. Lines: ----, baseline; — control.
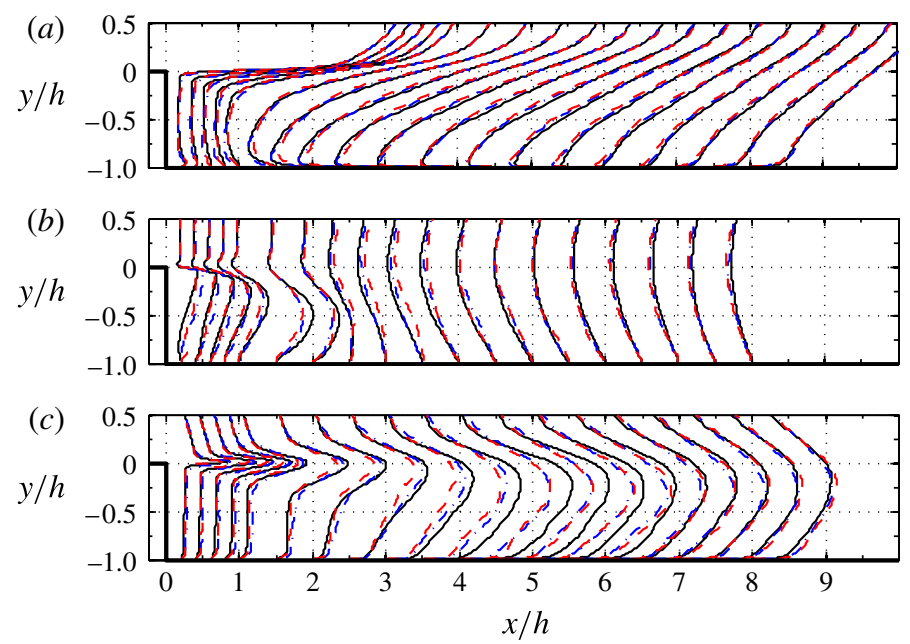

FIGURE 22. (Colour online) Flow characteristics downstream of separation for closed-loop forcing. (a) Time-averaged streamwise velocity profiles $\bar{U}$. (b) Time-averaged transverse velocity $\bar{V}$. (c) Turbulent kinetic energy $k=0.5\left(\overline{u^{\prime} u^{\prime}}+\overline{v^{\prime} v^{\prime}}+\overline{w^{\prime} w^{\prime}}\right)$. Lines: - , unforced flow; -...- , controller $K_{1}$ (actuator 1); - - - , controller $K_{2}$ (actuator 2).

both actuator locations. The reattachment lengths of the controlled flows are mildly increased compared to the baseline flow. For actuator $1, X_{r} / h=6.8$ while for actuator 2, $X_{r} / h=6.9$, compared to $X_{r} / h=6.2$ in the baseline case. This represents an increase of roughly $10 \%$ in $X_{r}$ for the controlled flows. It appears therefore that the recirculation length is not a useful indicator of the base pressure. In the laminar case it was shown that the base pressure can be increased via control leading to a stabilized near-wake and delayed reattachment. For the turbulent flow, open-loop forcing frequencies below $S t_{h}=0.1$ lead to a decrease in $X_{r}$ and an increase in $\overline{C_{P}}$ on the base, whereas the present feedback control strategy leads to a mild increase in 
(a)
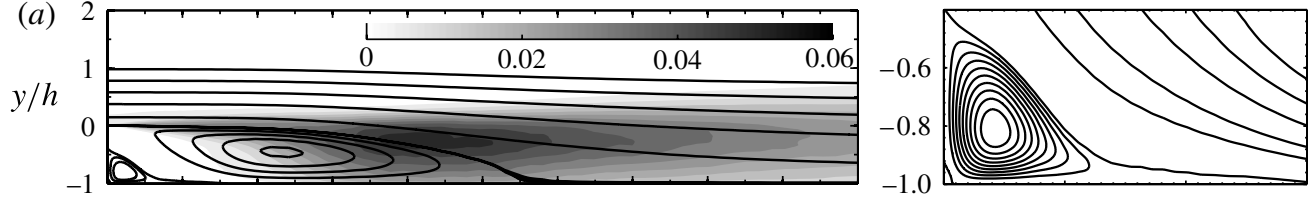

(b)
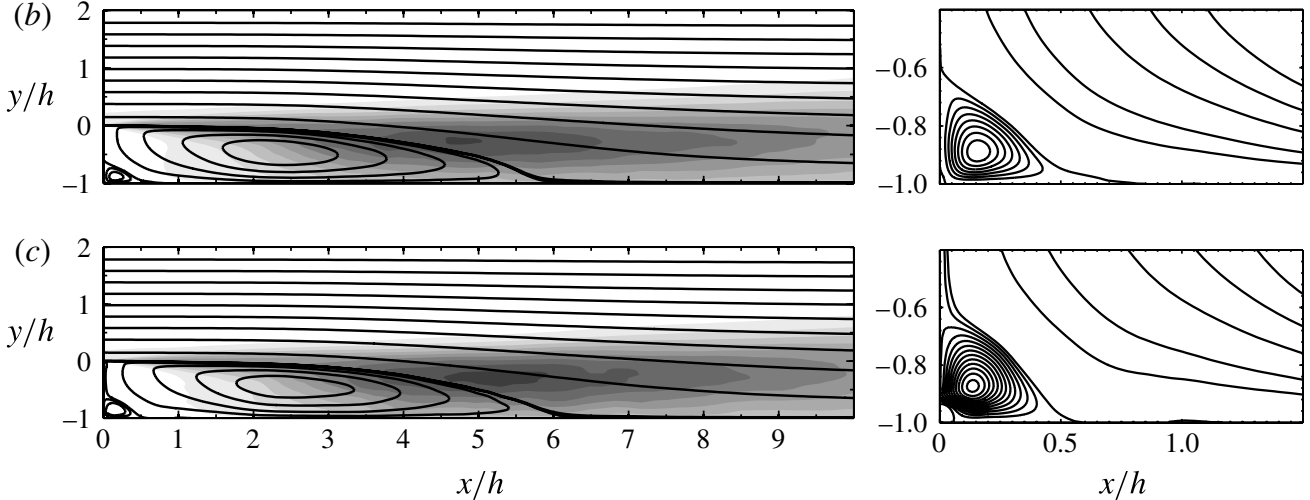

FIGURE 23. Line contours of the time-averaged streamfunction on colour contours of turbulent kinetic energy $k$. $(a)$ Baseline flow, $(b)$ controller $K_{1}$, and $(c)$ controller $K_{2}$. The right part of the figure shows a close-up on the base foot region.

$X_{r}$ and an increase in $\overline{C_{P}}$. Figure 23 also shows a magnified view of the secondary recirculation eddy sitting near the base foot. Both controllers reduce the size of this structure. This secondary recirculation bubble is the time-averaged view of bursts of high-momentum fluid, brought in from the reattachment region, impinging onto the base. It has been associated with the flapping mode (Spazzini et al. 2001), forming a feedback mechanism between the reattachment zone and the near-separation region. The reduction in pressure fluctuations attenuates this mechanism, which may be one of the causes of the increase in base pressure observed with the closed-loop control.

Instantaneous pressure contours in the plane $y=0$ are presented in figure 24 with and without control. Alternating spanwise structures of high and low pressure can be identified for the baseline and both controlled flows, but $K_{1}$ produces tighter and more 2D structures in the initial stage of the shear layer, before successive structures appear to merge. $K_{2}$ on the contrary leads to higher three-dimensionality and localized high-pressure spots are apparent.

A measure of the actuation cost is needed to compare the performance of the feedback control to the highest efficiency obtained with the open-loop forcing. We quantify the actuation cost with the momentum coefficient, $c_{\mu}=s U_{j, r m s}^{2} /\left(h U_{0}^{2}\right)$, where $s$ is the actuator width. We define the efficiency of an open-loop or closed-loop control scheme with the merit function $\mathscr{J}=\left(\overline{C_{P}}-\overline{C_{P 0}}\right) /\left(c_{\mu} \overline{C_{P 0}}\right)$. Table 1 gives the momentum coefficients and associated merit functions obtained with the best open-loop control (the one with the highest merit function) and with feedback control, for the two actuator locations considered. Considering actuator 1 , the highest merit function with open-loop forcing is achieved with an amplitude $A_{j}=0.1$ and forcing frequency $S t_{h}=0.05$. The corresponding momentum coefficient and mean pressure increase are $c_{\mu}=1.5 \times 10^{-4}$ and $24 \%$ respectively, which yields $\mathscr{J}=0.24 /\left(1.5 \times 10^{-4}\right)=1600$. The controller $K_{1}$ costs $c_{\mu}=1.2 \times 10^{-4}$ for a $20 \%$ increase in $\overline{C_{P}}$. Hence, its 
(a)

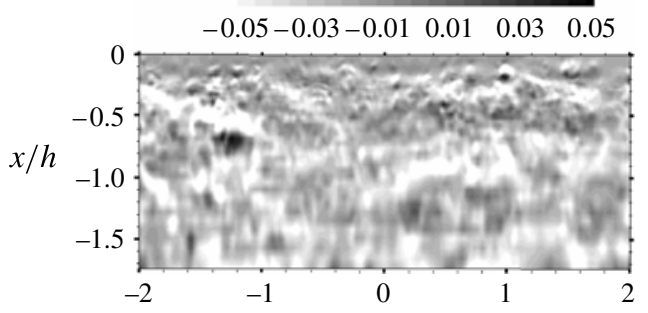

(b)

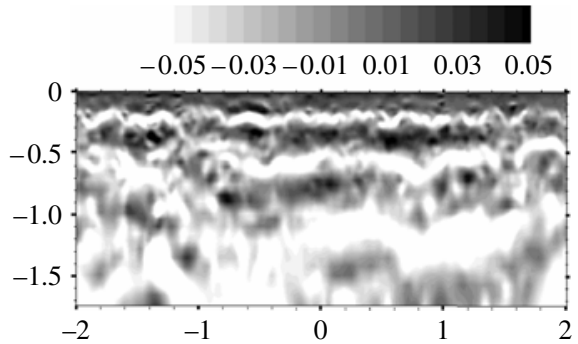

(c)

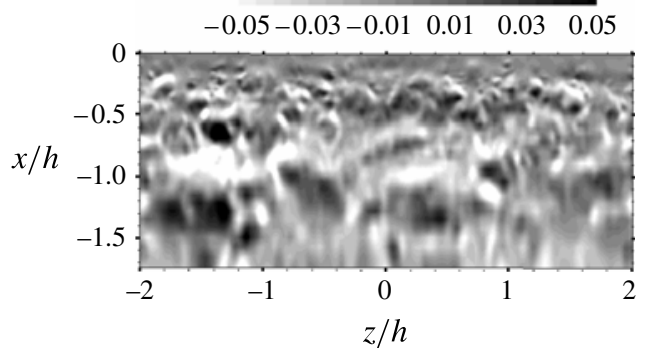

FIGURE 24. Instantaneous contours of pressure, $C_{P}$, in the plane $y=0$ downstream of separation. (a) Baseline flow, and $(b, c)$ the controlled flows for the first and second controllers $K_{1}$ and $K_{2}$, respectively.

\begin{tabular}{lccccc} 
& \multicolumn{2}{c}{ Actuator 1} & & \multicolumn{2}{c}{ Actuator 2} \\
\cline { 2 - 3 } \cline { 5 - 6 } Configuration & $A_{j}=0.1, S t_{h}=0.05$ & $K_{1}$ & & - & $K_{2}$ \\
$c_{\mu}$ & $1.5 \times 10^{-4}$ & $1.2 \times 10^{-4}$ & & - & $1.44 \times 10^{-4}$ \\
$\mathscr{J}$ & 1600 & 1667 & & 0 & 694
\end{tabular}

TABLE 1. Momentum coefficients $c_{\mu}$ and merit functions $\mathscr{J}$ for open-loop and closedloop control. For open-loop, the numbers shown correspond to the harmonic input with the highest merit function obtained.

merit function is $\mathscr{J}=\Delta \overline{C_{P}} / c_{\mu}=0.2 / 1.2 \times 10^{-4} \approx 1667$. Therefore, the feedback controller $K_{1}$ is more efficient than the corresponding open-loop forcing. Regarding the secondary actuator, no sensible pressure increase was measured in open-loop whilst the controller $K_{2}$ has a merit function $\mathscr{J}=694$, which leads us to conclude that the first actuation location is a more appropriate choice to increase the base pressure and hence reduce form drag. Furthermore, the closed-loop results above were obtained with little consideration for actuation cost. Investigations with optimal control tools will be considered in future work and are expected to reduce the momentum coefficient required in the closed-loop case.

\section{Conclusions}

This paper showed that linear feedback control can be used to increase the base pressure on a backward-facing step, and hence reduce the form drag of a body whose downstream end resembles a BFS flow. 
The method involved deriving a low-order model of the flow response via system identification using harmonic forcing. This forcing provided some physical insight into the flow dynamics. Notably it was observed that perturbing the flow close to the shedding frequency leads to a large decrease in base pressure, concomitant with increased turbulent kinetic energy in the near-separation region, while low-frequency forcing increases the mean pressure. The design of the feedback controller was based on the premise that reducing the magnitude of the base pressure fluctuations results in an increase in the time-averaged base pressure. Thus a simple linear controller was designed, based on the low-order model of the flow response, to reduce the amplitude of the fluctuations. In both the 2D laminar and 3D turbulent flow cases, the feedback controller achieved both a decrease in fluctuations and an increase in mean base pressure, using actuation velocities substantially lower than the free-stream velocity. In $2 \mathrm{D}$, the controllers stabilized the near-wake by pushing the unsteady reattachment region further downstream. After a transient period, the controllers were able to reduce their effort to the minimum required to prevent the redevelopment of the instability. In 3D, the feedback control extended the reattachment length by roughly $10 \%$ and reduced the turbulent kinetic energy in the shear layer. Both actuators were effective although actuator 1 outperformed actuator 2 with regard to the mean pressure increase in $\Omega_{3 D}$.

Comparison between two actuation locations and controllers in the 3D case revealed that the link between the base pressure fluctuations and the time-averaged base pressure is complex. Optimization using this approach may therefore present some difficulties, since a larger attenuation of the pressure fluctuations is not necessarily associated with a larger mean pressure increase. Future work will attempt to shed more light on this point. In addition, it was shown that there is no direct link between the reattachment length and the base pressure.

The closed-loop performance inherently depends on the open-loop system. The actuator location was notably observed to be a significant parameter. It was seen that the flow can be significantly modified by feedback with an actuator in an unusual location, near the base foot, even though the open-loop harmonic forcing does not show strong effects on the mean flow.

\section{Acknowledgements}

The authors gratefully acknowledge financial support from the Engineering and Physical Sciences Research Council. They also wish to thank S. Burbidge for time allocated on the Imperial High Performance Computing service and E. Brosco for the PIV data.

\section{REFERENCES}

Aram, E., Mittal, R. \& Cattafesta, L. 2010 Simple representations of zero-net-mass-flux jets in grazing flow for flow-control simulations. Intl J. Flow Control 2 (2), 109-125.

Bhattacharjee, S., Scheelke, B. \& Troutt, T. R. 1986 Modifications of vortex interactions in a reattaching separated flow. AIAA J. 24 (4), 623-629.

BRADShAW, P. \& WONG, F. Y. F. 1972 The reattachment and relaxation of a turbulent shear layer. J. Fluid Mech. 52, 113-135.

Brosco, E. 2011 Experimental investigation on separation control by high frequency forcing. $\mathrm{PhD}$ thesis, University of Rome, La Sapienza.

Cattafesta, L. N. \& Sheplak, M. 2011 Actuators for active flow control. Annu. Rev. Fluid Mech. 43, 247-272. 
Cattafesta, L. N., Song, Q., Williams, D. R., Rowley, C. W. \& Alvi, F. S. 2008 Active control of flow-induced cavity oscillations. Prog. Aerosp. Sci. 44, 479-502.

Chun, K. B. \& SUnG, H. J. 1996 Control of turbulent separated flow over a backward-facing step by local forcing. Exp. Fluids 25, 133-142.

Cohen, K., Siegel, S. \& Mclaughlin, T. 2006 A heuristic approach to effective sensor placement for modelling of a cylinder wake. Comput. Fluids 35, 103-120.

Dandois, J., GARnier, E. \& SAgaut, P. 2007 Numerical simulation of active separation control by a synthetic jet. J. Fluid Mech. 574, 25-58.

DejoAn, A. \& Leschziner, M. A. 2004 Large eddy simulation of periodically perturbed separated flow over a backward-facing step. Intl J. Heat Transfer Fluid Flow 25, 581-592.

Dowling, A. P. \& Morgans, A. S. 2005 Feedback control of combustion oscillations. Annu. Rev. Fluid Mech. 37, 151-182.

Driver, D. M. \& Seegmiller, H. L. 1985 Features of a reattaching turbulent shear layer in divergent channel flow. AIAA J. 23 (2), 163-171.

EAton, J. K. \& Johnston, J. P. 1981 A review of research on subsonic turbulent flow reattachment. AIAA J. 19 (9), 1093-1100.

ENGLAR, R. J. 2000 Development of pneumatic aerodynamic devices to improve the performance, economics, and safety of heavy vehicles. SAE Technical Paper Series, 2000-01-2208.

Fishpool, G. M. \& Leschziner, M. A. 2009 Stability bounds for explicit fractional-step schemes for the Navier-Stokes equations at high Reynolds number. Comput. Fluids 38, 1289-1298.

Gad-el Hak, M., Pollard, A. \& Bonnet, J.-P. 1998 Flow Control, Fundamentals and Practices. Springer.

Gelb, A. \& VAnder Velde, W. E. 1968 Multiple-Input Describing Functions and Nonlinear System Design. McGraw-Hill.

HASAN, M. A. Z. 1992 The flow over a backward-facing step under controlled perturbation: laminar separation. J. Fluid Mech. 238, 73-96.

HASAN, M. A. Z. \& KHAN, A. S. 1992 On the instability characteristics of a reattaching shear layer with nonlaminar separation. Intl J. Heat Transfer Fluid Flow 13 (3), 224-231.

HeEnan, A. F. \& MorRison, J. F. 1998 Passive control of pressure fluctuations generated by separated flow. AIAA J. 36 (6), 1014-1022.

HENNing, L. \& KING, R. 2005 Drag reduction by closed-loop control of a separated flow over a bluff body with a blunt trailing edge. In Proceedings of the 44th IEEE Conference on Decision and Control, and the European Control Conference 2005, pp. 494-499. IEEE.

Henning, L. \& KING, R. 2007 Robust multivariable closed-loop control of a turbulent backward-facing step flow. J. Aircraft 44 (1), 201-208.

ILLINGWORTH, S. J. 2009 Feedback control of oscillations in combustion and cavity flows. PhD thesis, Magdalene College, University of Cambridge.

Jimenez, J., Hoyas, S., Simens, M. P. \& Mizuno, Y. 2010 Turbulent boundary layers and channels at moderate Reynolds numbers. J. Fluid Mech. 657, 335-360.

JUANG, J.-N. \& PAPPA, R. S. 1985 An eigensystem realization algorithm for modal parameter identification and model reduction. J. Guid. Control Dyn. 8 (5), 620-627.

Kim, J., Hahn, S., Kim, J., Lee, D., Choi, J., JeOn, W. \& Choi, H. 2004 Active control of turbulent flow over a model vehicle for drag reduction. J. Turbul. 5, 019.

Kim, K., Kerr, M., Beskok, A. \& JayAsuriya, S. 2006 Frequency-domain based feedback control of flow separation using synthetic jets. In Proceedings of the 2006 American Control Conference, pp. 5318-5323. IEEE.

LARDAT, R. \& LESCHZINER, M. A. 1998 A Navier-Stokes solver for LES on parallel computers. Tech. Rep. Department of Mechanical Engineering, UMIST.

LE, H., Moin, P. \& KIM, J. 1997 Direct numerical simulation of turbulent flow over a backward-facing step. J. Fluid Mech. 330, 349-374.

Leschziner, M. A. \& Lardeau, S. 2011 Simulation of slot and round synthetic jets in the context of boundary layer separation. Phil. Trans. R. Soc. A 369, 1495-1512.

LJUNG, L. 1999 System Identification: Theory for the User. Prentice Hall.

Lund, T. S., WU, X. \& SQuiRES, K. D. 1998 Generation of turbulent inflow data for spatially-developing boundary layer simulations. J. Comput. Phys. 140, 233-258. 
Ma, Z., Ahuja, S. \& Rowley, C. W. 2009 Reduced order models for control of fluids using the eigensystem realization algorithm. Theor. Comput. Fluid Dyn. 25, 233-247.

McFarlane, D. C. \& Glover, K. 1989 Robust Controller Design Using Normalized Coprime Factor Plant Descriptions. Springer.

Modi, V. J., Hill, S. ST. \& Yokomizo, T. 1995 Drag reduction of trucks through boundary-layer control. J. Wind Engng Ind. Aerodyn. 54/55, 583-594.

Nicoud, F. \& Ducros, F. 1999 Subgrid-scale stress modelling based on the square of the velocity gradient tensor. Flow Turbul. Combust. 62, 183, 200.

Park, H., Lee, D., Jeon, W.-P., Hahn, S., Kim, J., Kim, J., Choi, J. \& Choi, H. 2006 Drag reduction in flow over a two-dimensional bluff body with a blunt trailing edge using a new passive device. J. Fluid Mech. 563, 389-414.

Pastoor, M., Henning, L., Noack, B. R., King, R. \& Tadmor, G. 2008 Feedback shear layer control for bluff body drag reduction. J. Fluid Mech. 608, 161-196.

QubAIn, A. 2009 Active control of a turbulent bluff body wake. PhD thesis, Imperial College London.

Roshko, A. 1954 On the drag and shedding frequency of two-dimensional bluff bodies. Tech. Rep. National Advisory Committee for Aeronautics.

Seifert, A., Stalnov, O., Sperber, D., Arwatz, G., Palei, V., David, S., Dayan, I. \& FONO, I. 2009 Large trucks drag reduction using active flow control. In The Aerodynamics of Heavy Vehicles II: Trucks, Buses, and Trains (ed. F. Browand, R. McCallen \& J. Ross). Lecture Notes in Applied and Computational Mechanics, vol. 41, pp. 115-133. Springer.

Siegel, S., Cohen, K. \& MCLaughlin, T. 2006 Numerical simulations of a feedback-controlled circular cylinder wake. AIAA J. 44 (6), 1266-1276.

Simpson, R. L. 1989 Turbulent boundary-layer separation. Annu. Rev. Fluid Mech. 21, 205-234.

Spazzini, P. G., Iuso, G., Onorato, M., Zurlo, N. \& Di CicCA, G. M. 2001 Unsteady behaviour of back-facing step flow. Exp. Fluids 30, 551-561.

Stalnov, O., Fono, I. \& SEIFERT, A. 2011 Closed-loop bluff-body wake stabilization via fluidic excitation. Theor. Comput. Fluid Dyn. 25, 209-219.

TAnner, M. 1972 A method for reducing the base drag of wings with blunt trailing edge. Aeronaut. Q. 23, 15-23.

Temmerman, L. 2004 Large eddy simulation of separating flows from curved surfaces. PhD thesis, University of London.

Troutt, T. R., Scheelke, B. \& Norman, T. R. 1984 Organized structures in a reattaching separated flow field. J. Fluid Mech. 143, 413-427.

URubA, V., JonÁš, P. \& MAZur, O. 2007 Control of a channel-flow behind a backward-facing step by suction/blowing. Intl J. Heat Transfer Fluid Flow 28, 665-672.

Wengle, H., Huppertz, A., B ̈̈rwolff, G. \& JAnke, G. 2001 The manipulated transitional backward-facing step flow: an experimental and direct numerical simulation investigation. Eur. J. Mech. B 20 (1), 25-46.

Wood, C. J. 1967 Visualization of an incompressible wake with base bleed. J. Fluid Mech. 29 (2), 259-272.

Yoshioka, S., Овi, S. \& MASUdA, S. 2001 Organized vortex motion in periodically perturbed turbulent separated flow over a backward-facing step. Intl J. Heat Transfer Fluid Flow 22, 301-307. 